Preprint typeset in JHEP style - HYPER VERSION

SHEP 02-22

\title{
A proposal for a manifestly gauge invariant and universal calculus in Yang-Mills theory
}

\author{
Stefano Arnone, Antonio Gatti and Tim R. Morris \\ Department of Physics and Astronomy, University of Southampton \\ Highfield, Southampton SO17 1BJ, U.K. \\ E-mails: sa@hep.phys.soton.ac.uk, gatti@hep.phys.soton.ac.uk, \\ T.R.Morris@soton.ac.uk
}

\begin{abstract}
We uncover a method of calculation that proceeds at every step without fixing the gauge or specifying details of the regularisation scheme. Results are obtained by iterated use of integration by parts and gauge invariance identities. The initial stages can even be computed diagrammatically. The method is formulated within the framework of an exact renormalization group for $S U(N)$ Yang-Mills gauge theory, incorporating an effective cutoff through a manifest spontaneously broken $S U(N \mid N)$ gauge invariance. We demonstrate the technique with a compact calculation of the one-loop beta function, achieving a manifestly universal result, and without gauge fixing, for the first time at finite $N$.
\end{abstract}




\section{Contents}

1. Introduction 2

1.1 Overview 1

2. Preliminary comments 5

2.1 The regularisation 5

2.2 Necessary properties of the exact RG and their interpretation 8

2.3 Supergauge invariance and functional derivatives 10

2.4 Covariantisation 12

2.5 Decoration with $\mathcal{C} \quad 14$

2.6 Superfield expansion 15

3. A manifestly $S U(N \mid N)$ gauge invariant exact RG

3.1 Supersowing and supersplitting in the $\mathcal{A}$ sector 20

3.2 Diagrammatic interpretation 22

3.3 After spontaneous breaking 23

3.4 (Un)broken gauge invariance 24

4. Seed action two-point vertices 26

5. The kernels 27

6. The integrated kernels 29

6.1 Five dimensions in the fermionic sector 30

7. Guaranteeing universality of $\beta_{1}\left(\right.$ and $\left.\beta_{2}\right)$

7.1 Ensuring no running couplings at tree level 33

8. The calculation 35

8.1 Diagrammatic analysis 36

8.2 The total $\Lambda$ derivative contribution 44

8.3 The gauge remainders 46

8.4 The $c_{0}^{\prime}$ terms 48

9. Conclusions 48 


\section{Introduction}

It need hardly be stressed that there is a clear need for a better non-perturbative understanding of quantum field theory. Examples can be given from all domains where quantum field theory is applicable: from phase transitions in early universe cosmology, quantum gravity, QCD, through to high temperature superconductivity, to mention just a few.

The exact Renormalization Group (RG) 2, 25], the continuum version of a Wilsonian $\mathrm{RG}$, provides a powerful framework for considering non-perturbative analytic approximations to quantum field theories [3, 4, 5, 8, 26]. This follows from the fact that solutions of the corresponding flow equations, i.e. the Wilsonian effective action, can be found directly in terms of renormalized quantities, that all physics (e.g. Green functions) can be extracted from this, and that renormalizability is trivially preserved in almost any approximation [4, 5].

Central to many non-perturbative problems, including the examples quoted above, is the presence of gauge invariance. (This local invariance can be accepted as either fundamental or, e.g. in the case of high $T_{c}$, effective [6].) However the introduction of a real ${ }^{1}$ effective cutoff $\Lambda$, a crucial step in the definition of a Wilsonian RG, typically breaks this gauge invariance [i].

Fortunately it is possible to formulate more general exact RGs [8], which are gauge invariant [9, 10, 11, 12]. A wonderful extra benefit in this generalised framework is that calculations can proceed with manifest gauge invariance preserved at every stage [9, 10, 11, 12]. There is thus no need for gauge fixing and the corresponding ghosts. The challenging non-perturbative problem of Gribov copies [13], is thus entirely avoided. ${ }^{2}$ Even at the perturbative level the full power and beauty of gauge invariance then shines through. Unlike BRST transformations, gauge transformations are at most linear in the quantum fields and thus are not deformed at the quantum level. For a non-Abelian gauge group, the connection in the covariant derivative thus remains dimension one and is unrenormalized. If the coupling is scaled out of the connection, no wave function renormalization is possible for the gauge field [9, 10, 11, 12]. Only the coupling renormalizes. The usually naïve assumption that the effective action is built only from gauge invariant combinations of the covariant derivative is here true even at the quantum level; all vertices are subject to simple strong constraints - the so-called "naïve Ward identities" that follow from exact gauge invariance.

In order to formulate such a gauge invariant exact $R G$, we need to incorporate a gauge invariant real cutoff $\Lambda$. Moreover, this has to appear in a way that can be naturally incorporated in the effective action framework [9, 11, 12]. We use the solution given in ref. [16], which provides a regularisation for $S U(N)$ Yang-Mills theory in $D \leq 4$ spacetime dimensions, and when $N=\infty$, in any dimension. This is implemented by embedding the Yang-Mills theory in a $S U(N \mid N)$ gauge theory, regularised by covariant higher derivatives. The $S U(N \mid N)$ gauge theory is then spontaneously broken in the fermionic directions, at the same scale $\Lambda$, with the resulting heavy fields playing the rôle of gauge invariant Pauli-

\footnotetext{
${ }^{1}$ as opposed to e.g. analytic continuation of perturbative amplitudes in dimensional regularisation

${ }^{2}$ Gribov problems are known to result in an erroneous answer for covariant gauges [14, 15.
} 
Villars fields 17]. (Actually this corresponds to regulating a $S U(N) \times S U(N)$ Yang-Mills theory, but the non-unitary second copy decouples in the continuum limit [16].)

The work presented in this paper extends previously published results in a number of significant ways. The flow equation in ref. [11] was developed intuitively from the bottom up without the author being aware of the underlying $S U(N \mid N)$ structure [9]. In contrast, the present flow equation is very simple and beautiful in its conception, its form being tightly constrained by the manifest invariance under the spontaneously broken local $S U(N \mid N)$. The earlier flow equation in ref. [11] was regularised only to one loop (and then only for external gauge fields). Here the gauge invariant regularisation is complete, working to all orders in perturbation theory. The formulation given in ref. [11] was restricted to $N=\infty$ (again as consequence of regularisation limitations). The present formulation makes sense also at finite $N$.

And last but by no means least, a powerful computational technique for working within this framework, is developed, building on the insights gained from ref. [18]. In all realisations of the Wilsonian RG there is an unavoidable freedom in the construction, equivalent to regularisation scheme dependence, but especially deeply embedded [8]. Thus for example any version of the exact $R G$ receives its very definition in part by specifying a cutoff function $c$. This redundancy is magnified in the case of these gauge invariant exact RGs, because the regularisation requires a further cutoff function $\tilde{c}$, and the requirement of gauge invariance forces the introduction of further choices, to a large extent arbitrary, namely the covariantisation(s) ${ }^{3}$ and the "seed" interactions. Nevertheless, physical quantities must be independent of these choices. Providing that we limit ourselves to controlled expansions (e.g. those in weak coupling, strong coupling, $1 / N, 1 / D$ etc. ), the same must be true of the approximations.

There ought then to be a way of computing these results without having to specify the above choices of regularisation scheme. We uncover just such a method. The large redundancy in the regularisation scheme is turned to our advantage, furnishing a guide to streamlined computation of universal quantities. Thus in the method, we are forbidden from 'looking inside' any vertices (of the the seed action or covariantised kernels but also of the undifferentiated Wilsonian effective action). The initial stages of the calculation are then so constrained that they can be effectively performed diagrammatically.

Central to the method are integrated exact RG kernels which play the rôle of regularised propagators, specifically by being the inverse of the corresponding two-point vertices. For the gauge fields, since gauge invariance is preserved, these inverses do not exist. Instead the integrated kernels are inverses only in the transverse space, leaving longitudinal remainders that generate gauge transformations.

These 'effective propagators' are introduced by integrating by parts with respect to $\Lambda$, resulting in differentials of the Wilsonian effective action. These latter are evaluated via their flow equations, after which gauge invariance identities are used to evaluate further, where possible. This procedure is iterated until there are no terms left that depend on the choice of covariantisation or seed interactions. It is then straightforward to cast the

\footnotetext{
${ }^{3}$ Different parts of the flow equation can even have different covariantisations.
} 
remaining terms as total derivatives in momentum space or otherwise show them to be universal.

Although we apply the method here only to the computation of the one-loop $\beta$ function of $S U(N)$ Yang-Mills theory, we believe the procedure to be of general applicability. In fact our aim is to apply these ideas to the non-perturbative domain. As already mentioned, exact RG equations are ideally suited for this. It is important to note in this context that our gauge invariant exact RG equation and the regularisation it embodies do not require perturbation theory for their definition.

A necessary step is to thoroughly test and understand the framework in the perturbative domain. For calculations at two loops and beyond, it is helpful to augment the present flow equation. A full report of this investigation however is left for the future [1].

\subsection{Overview}

Most of the present paper, up to sec. 8, is concerned with setting up and justifying the formalism. Specifically, we start in sec. 2.1 with a review of the regularisation [16], and some of its novelties, adapting it to the exact RG constructed in this paper. Although we leave many elements of the exact RG unspecified, using this freedom to guide the calculation and display universality, there are a number of basic restrictions needed on the set of exact RGs we allow. Sec. 2.2 sets out the general properties we require, and their consequences and interpretation in the present case. Sec. 2.3 deals with a particular novelty that arises in the transformation of the $S U(N \mid N)$ supergauge field functional derivative, which in turn leads to a further constraint on the form of the exact RG. Secs. 2.4 and 2.5 spell out the restrictions placed on the form of supergauge covariantization and further decoration of momentum space kernels, and introduce the general notation used to define the resulting vertices. In sec. 2.6, we introduce the corresponding notation for action vertices, and the form the notation takes after spontaneous supersymmetry breaking.

In sec. 3 we introduce the flow equation, defining various elements and developing some of its properties. In particular we prove its supergauge invariance, and in sec. 3.1 prove a closely related property that leads to a powerful diagrammatic incorporation of the supergauge algebra, as is explained in sec. 3.2. From sec. 3.3 onwards we work in the spontaneously broken theory, sec. 3.4 containing the centrally important resulting relations between vertices, the so-called naïve Ward identities, both for the remaining $S U(N) \times$ $S U(N)$ bosonic gauge invariance, but also for the broken fermionic gauge invariances.

In sec. 4 , we use general arguments to determine the form of the classical effective action two-point vertices. These are used to determine the kernels in sec. 5, and thus the integrated kernels in sec. 6. Most importantly, we show how these behave as effective propagators up to gauge remainder terms. Together with sec. 3.4, these provide the essential properties behind the 'calculus' that follows. These properties are seen clearly in the broken fermionic sector, if the fermionic parts are combined into a $D+1$ dimensional vector as in sec. 6.1, a notation we then adopt for the rest of the paper. Sec. 0 explains precisely when one can expect to get a universal result for the first two coefficients of the Yang-Mills $\beta$ function. Although this is standard, the universality is actually violated without the further restrictions that 
are introduced in sec. 7.1, a novel consequence of Pauli-Villars regularisation in an exact RG framework [9, 11].

Finally, in sec. 8, we set out the calculation, with sec. 8.1 in particular containing the main iterative diagrammatic procedure, and sec. 8.2 the heart of the calculation from the physics point of view. In sec. 9 we summarise and draw our conclusions.

\section{Preliminary comments}

Throughout the paper we work in Euclidean space of dimension four. We can formulate everything in general dimension $D$, and strictly speaking should, since the limit $D \rightarrow 4$ is necessary to rigorously define the regularisation [16]. However, as we will show, for the calculation of terms such as the one-loop $\beta$ function in $S U(N)$ Yang-Mills, we do not need to pay attention to this subtlety. Therefore for simplicity of exposition we will set $D=4$ in this paper, leaving the full generality until ref. [1].

\subsection{The regularisation}

Instead of working just with the $S U(N)$ gauge field, which we write as $A_{\mu}^{1}(x) \equiv A_{a \mu}^{1} \tau_{1}^{a}$, where $\tau_{1}^{a}$ are the $S U(N)$ generators orthonormalised to $\operatorname{tr}\left(\tau_{1}^{a} \tau_{1}^{b}\right)=\delta^{a b} / 2$, we embed it in a $S U(N \mid N)$ supergauge field [16:

$$
\mathcal{A}_{\mu}=\mathcal{A}_{\mu}^{0} 11+\left(\begin{array}{ll}
A_{\mu}^{1} & B_{\mu} \\
\bar{B}_{\mu} & A_{\mu}^{2}
\end{array}\right) .
$$

Here we have written $\mathcal{A}$ as an element of the $S U(N \mid N)$ Lie superalgebra, using the defining representation, i.e. as a supermatrix with bosonic block diagonal terms $A^{i}$ and fermionic block off-diagonals $B$ and $\bar{B}$, together with the central term $\mathcal{A}^{0} 1$. As required by $S U(N \mid N)$, the supermatrix (and thus also $\mathcal{A}$ ) is supertraceless, i.e. $\operatorname{tr} A^{1}-\operatorname{tr} A^{2}=0$. This excludes in particular

$$
\sigma \equiv \sigma_{3}=\left(\begin{array}{cc}
1 & 0 \\
0 & -11
\end{array}\right)
$$

from the Lie algebra. The supermatrix is in addition also traceless, the trace having been parametrised by $\mathcal{A}^{0}$. Equivalently, we can introduce a complete set of traceless and supertraceless generators $T_{A}$ (normalised as in ref. [16]) and thus expand $\mathcal{A}$ as

$$
\mathcal{A}_{\mu}=\mathcal{A}_{\mu}^{0} 11+\mathcal{A}_{\mu}^{A} T_{A} \text {. }
$$

The $B$ fields are wrong statistics gauge fields. They will be given a mass of order the cutoff $\Lambda$. The supergroup $S U(N \mid N)$ has $S U(N) \times S U(N) \times U(1)$ as its bosonic subgroup. $A_{\mu}^{2}(x) \equiv A_{a \mu}^{2} \tau_{2}^{a}$ is the gauge field for the second $S U(N)$, and $\mathcal{A}^{0}$ is the $U(1)$ connection. Interactions are built via commutators, using the covariant derivative:

$$
\nabla_{\mu}=\partial_{\mu}-i \mathcal{A}_{\mu}
$$

thus the superfield strength is given by $\mathcal{F}_{\mu \nu}=i\left[\nabla_{\mu}, \nabla_{\nu}\right]$. The kinetic term will be regularised by higher derivatives which thus take the form:

$$
\operatorname{str} \mathcal{F}_{\mu \nu}\left(\frac{\nabla}{\Lambda}\right)^{n} \cdot \mathcal{F}_{\mu \nu}
$$


(where the dot means $\nabla$ acts by commutation. In practice we will add the higher derivatives as a power series with coefficients determined by a cutoff function $c$.) The supertrace, which is necessary to ensure $S U(N \mid N)$ invariance, forces the kinetic term for $A^{2}$ to have wrong sign action, leading to negative norms in its Fock space [16].

As can be seen from (2.3), $\mathcal{A}^{0}$ does not appear in the kinetic term. Providing the interactions can be written as $\operatorname{str}(\mathcal{A} \times$ commutators $), \mathcal{A}^{0}$ will not appear anywhere in the action. More generally we will need to impose its non-appearance as a constraint, since otherwise $\mathcal{A}^{0}$ has interactions but no kinetic term and thus acts as a Lagrange multiplier. This would result in a non-linear constraint on the theory, which does not look promising for its use as a regularisation method for the original $S U(N)$ Yang-Mills.

On the other hand, if the constraint is satisfied, $\mathcal{A}^{0}$ is then protected from appearing by a local "no- $\mathcal{A}^{0}$ " shift symmetry: $\delta \mathcal{A}_{\mu}^{0}(x)=\lambda_{\mu}(x)$, which implies in particular that $\mathcal{A}^{0}$ has no degrees of freedom. Together with supergauge invariance the theory is then invariant under

$$
\delta \mathcal{A}_{\mu}=\nabla_{\mu} \cdot \Omega+\lambda_{\mu} \mathbb{1}
$$

(where the supermatrix $\Omega(x)$ is in the $S U(N \mid N)$ Lie algebra). The effect of the no$\mathcal{A}^{0}$ symmetry is to dynamically define the gauge group as the quotient $S U^{\prime}(N \mid N)=$ $S U(N \mid N) / U(1)$, in which Lie group elements are identified modulo addition of an arbitrary multiple of 1 .

An alternative and equivalent formulation [16] is to pick coset representatives, which can for example be taken to be traceless, so that $\mathcal{A}^{0}$ is set to zero, and thus discarded. (This is the strategy used in ref. [19] to define a $S U^{\prime}(N \mid N)$ sigma model. Incidentally this paper contains arguments for finiteness of these models which are similar to those given by us for $S U(N \mid N)$ gauge theory [16]. $\left.{ }^{4}\right)$ In this reduced representation, eq. (2.6) is replaced by Bars' bracket [20]:

$$
\delta \mathcal{A}_{\mu}=\left[\nabla_{\mu}, \Omega\right]^{*} \equiv\left[\nabla_{\mu}, \Omega\right]-\frac{1}{2 N} \operatorname{tr}\left[\nabla_{\mu}, \Omega\right] .
$$

The ${ }^{*}$ bracket replaces the commutator as a representation of the Lie product so in particular $\mathcal{F}_{\mu \nu}=i\left[\nabla_{\mu}, \nabla_{\nu}\right]^{*}[16]$.

The lowest dimension interaction that violates no- $\mathcal{A}^{0}$ symmetry contains four superfield strengths, for example:

$$
\operatorname{str}\left(\mathcal{F}_{\mu \nu}\right)^{2}\left(\mathcal{F}_{\lambda \sigma}\right)^{2}
$$

Such terms are not invariant under the 'Bars"' (2.7), either. Since (2.8) is already irrelevant, no- $\mathcal{A}^{0}$ symmetry is automatic for the conventional supergauge invariant bare action of ref. [16]. Here there is no such bare action, and interactions are generated by a largely unspecified exact RG, so we need to impose no- $\mathcal{A}^{0}$ as an extra constraint.

We introduce a superscalar field

$$
\mathcal{C}=\left(\begin{array}{cc}
C^{1} & D \\
\bar{D} & C^{2}
\end{array}\right)
$$

\footnotetext{
${ }^{4}$ We thank Hugh Osborn for drawing our attention to this paper
} 
in the fundamental $\otimes$ its complex conjugate representation, equivalently as a matrix in the defining representation of $U(N \mid N)$ [16]. Under supergauge transformations

$$
\delta \mathcal{C}=-i[\mathcal{C}, \Omega] .
$$

In the Bars* representation we do not replace this by a *bracket, since commutators are necessary for powers of $\mathcal{C}$ (appearing in its potential) to transform covariantly [16]. However, as in ref. [16], since working with the full cosets seems more elegant, we will employ (2.6) and the full representation in this paper.

We will arrange for $\mathcal{C}$ to develop a vacuum expectation value along the $\sigma$ direction, so that classically $\langle\mathcal{C}\rangle=\Lambda \sigma .^{5}$ This spontaneously breaks the $S U(N \mid N)$ gauge invariance down to its $S U(N) \times S U(N) \times U(1)$ bosonic subgroup and provides the fermionic fields $B$ and $D$ with masses of order $\Lambda$. In unitary gauge, the Goldstone mode $D$ is eaten by $B$. Since we will not fix the $S U(N \mid N)$ invariance, they instead gauge transform into each other and propagate as a composite unit (see sec. 6.1). We arrange for the remaining 'Higgs' fields $C^{i}$ also to have masses of order $\Lambda$.

In ref. [16], we proved by conventional methods that if the kinetic term of $\mathcal{A}$ is supplied with covariant higher derivatives (parametrised by the cutoff function $c$ ) enhancing its high momentum behaviour by a factor $c^{-1} \sim p^{2 r} / \Lambda^{2 r}$, and the kinetic term of $\mathcal{C}$ has its high momentum behaviour similarly enhanced by $\tilde{c}^{-1} \sim p^{2 \tilde{r}} / \Lambda^{2 \tilde{r}}$, then providing

$$
r-\tilde{r}>1 \text { and } \tilde{r}>1
$$

all amplitudes are ultraviolet finite to all orders of perturbation theory. Since the underlying theory is renormalizable, the Appelquist-Carazzone theorem implies that at energies much lower than the cutoff $\Lambda$, the remaining massless fields $A^{1}$ and $A^{2}$ decouple. In this way, we can use this framework as a regularisation of the original $S U(N)$ Yang-Mills theory carried by $A^{1}$.

In brief, the reasons for the above facts are as follows. Providing eqs. (2.11) hold, all divergences are superficially regularised by the covariant higher derivatives, except for some 'remainders' of one-loop graphs with only $\mathcal{A}$ fields as external legs and only four or less of these legs. These remainders form a symmetric phase contribution, in the sense that the superficially divergent interactions between $\mathcal{C}$ and $\mathcal{A}$ are just those that come from $\mathcal{C}$ 's covariant higher derivative kinetic term, whilst all terms containing a $\sigma$ from the breaking are already ultraviolet finite by power counting. For three or less external $\mathcal{A}$ legs the remainders vanish by the supertrace mechanism: the fact that in the unbroken theory, the resultant terms contain $\operatorname{str} \mathcal{A}=0$ or $\operatorname{str} \mathbb{1}=0$. By manifest gauge invariance, the four point $\mathcal{A}$ remainder is then actually totally transverse, which implies that it is already finite by power counting.

The decoupling of $A^{1}$ and $A^{2}$ follows from the unbroken local $S U(N) \times S U(N)$ invariance since the lowest dimension effective interaction

$$
\frac{1}{\Lambda^{4}} \operatorname{tr}\left(F_{\mu \nu}^{1}\right)^{2} \operatorname{tr}\left(F_{\mu \nu}^{2}\right)^{2}
$$

\footnotetext{
${ }^{5}$ Later however we will use an unconventional normalisation for $\mathcal{C}$.
} 
is already irrelevant [16, 21].

Actually, there are a number of differences between the treatment we give here and that of ref. [16]. Since ref. [16] followed a conventional treatment, gauge fixing and ghosts were introduced, with a corresponding higher derivative regularisation for them; longitudinal parts of the four point $\mathcal{A}$ vertex were then related to ghost vertices using the Lee ZinnJustin identities, which were separately proved to be finite. Also, a specific form of bare action and covariantisation was chosen.

Here we do not fix the gauge and the regularisation scheme is much more general. As well as not specifying the covariantisation or the bare action (see below) there is anyway much more freedom in introducing interactions via the flow equation. We shall not here supply a rigorous proof that up to appropriate restrictions, the flow equation is finite. Since we never have to specify the details, we only need to assume that this is true for at least one choice. However, we take care that the scheme as described above is qualitatively correctly implemented. Where we do have to explicitly compare terms we can use (2.11) as a guide, thus for example we do find that our final expression for $\beta_{1}$ is properly regulated. (However, it should be borne in mind that at intermediate stages our vertices have much more freedom in their momentum dependence than that implied by the bare action in ref. [16]. Additionally, cutoff functions with non-power law asymptotics, for example exponential, could also be used. ${ }^{6}$ ) In practice, it is easy to see at one loop that the high energy cancellations are occurring as expected.

\subsection{Necessary properties of the exact RG and their interpretation}

The extra fields we have added form a necessary part of the regularisation structure. We gain an interpretation of these fields at the effective level by imagining integrating out the heavy fields $B, C$ and $D$ at some scale $\Lambda$. The result would be an effective action containing only the unbroken gauge fields $A^{i}$, but it is not well defined because it is not finite. In particular, the one-loop determinant formed from integrating out the heavy fields is necessarily divergent: the divergences are there to cancel those left by the one-loop hole in the remaining covariant higher derivative regularisation [22] of the $S U(N) \times S U(N)$ Yang Mills theory, in a similar way to that done explicitly in gauge invariant Pauli-Villars regularisation [17].

A gauge invariant exact RG description of gauge theory thus requires not only a well defined finite effective action but a separate measure term. The measure term is not itself finite, but can be represented by a well-defined finite addition to the effective action, if we accept the introduction of these auxiliary heavy fields.

Whilst this interpretation is reasonable, we nevertheless need to be sure that we are still only representing the original quantum field theory (here $S U(N)$ Yang-Mills). This demand is especially pertinent in (but not restricted to) the case where there are extra regulator fields, particularly here $A^{2}$ which remains massless and in this effective description only decouples at momenta much less than $\Lambda$. More generally, even if there are only physical fields in the effective action, we need to be sure that locality, an important property of

\footnotetext{
${ }^{6}$ The proof given in ref. [16] could also be easily extended to these cases.
} 
quantum field theory [23, 24], is properly incorporated. ${ }^{7}$ Note that $\Lambda$ is intended to be set at the energy scales of interest, which is why it makes sense to use the exact RG and solve for the effective action directly in renormalized terms (see e.g. ref. [5]). Indeed, to extract the physics (e.g. correlation functions etc. ) we will even want to take $\Lambda \rightarrow 0$ eventually [4, 包.

These demands are fulfilled implicitly through the $\Lambda \rightarrow \infty$ limit, providing some very general requirements on the exact $\mathrm{RG}$ are implemented, as we now explain.

Firstly, we require that all parts of the flow equation can be expanded in external momenta to any order, so that the solutions $S$ can also be required to have an all orders derivative expansion [10, 11, 5]. ${ }^{8}$ This 'quasilocality' requirement [10] is equivalent to the fundamental requirement of the Wilsonian RG that Kadanoff blocking take place only over a localised patch [2], i.e. here that each RG step, $\Lambda \mapsto \Lambda-\delta \Lambda$, be free from infrared singularities.

The flow equation is written only in terms of renormalized quantities at scale $\Lambda$. In fact, we require that the only explicit scale parameter that appears in the equations is the effective cutoff $\Lambda$. Again this is so that the same can be required of $S$ where it implements the concept of self-similar flow [27]. Here this amounts to a non-perturbative statement of renormalizability, i.e. existence of a continuum limit, equivalent to the requirement that $S$ lie on a renormalized trajectory [5]. This is clearer if we first scale to dimensionless quantities using the appropriate powers of $\Lambda$. Then, $S$ is required to have no dependence on $\Lambda$ at all except through its dependence on the running coupling(s) $g(\Lambda)$ [5].

Note that the $\Lambda \rightarrow \infty$ end of the renormalized trajectory, i.e. the perfect action 28. in the neighbourhood of the ultraviolet fixed point at $\Lambda=\infty$, amounts to our choice of bare action. Its precise form is not determined beforehand but as a result of solution of the exact RG, but it is constrained by choices in the flow equation. Since these choices are however here to a large extent unmade, we deal implicitly with an infinite class of perfect bare actions.

We require that the flow of the Boltzmann measure $\exp (-S)$ is a total functional derivative, i.e. for some generic fields $\phi$ :

$$
\Lambda \partial_{\Lambda} \mathrm{e}^{-S}=\frac{\delta}{\delta \phi}\left(\Psi \mathrm{e}^{-S}\right)
$$

(corresponding to the statement that each RG step is equivalent to an infinitesimal field redefinition $\phi \mapsto \phi+\Psi \delta \Lambda)$ [9, 8]. Importantly, this ensures that the partition function $\mathcal{Z}=\int \mathcal{D} \phi \exp (-S)$, and hence the physics derived from it, is invariant under the RG flow. Since we will solve the exact RG approximately, but by controlled expansion in a small quantity, this property is left undisturbed. Therefore we may use different scales $\Lambda$ at our convenience to interpret the computation.

For example, although locality is obscured in the Wilsonian effective action at any finite $\Lambda$, it is important to recognise that invariance of $\mathcal{Z}$ together with the existence of a derivative expansion and self-similar flow (viz. that the only explicit scale be $\Lambda$ ), ensure

\footnotetext{
${ }^{7}$ otherwise non-physical effects or the effects of other propagating fields, could be hidden in the vertices.

${ }^{8}$ Sharp cutoff realisations [25] are more subtle [4, 26] and will not be discussed here.
} 
that locality is implemented, since it is then an automatic property of the effective action as $\Lambda \rightarrow \infty$.

Similarly, it is as $\Lambda \rightarrow \infty$ that we confirm from the Wilsonian effective action that we are describing $S U(N)$ Yang-Mills theory: $B, C$ and $D$ really are infinitely massive, and in spacetime dimension four or less, $A^{2}$ is guaranteed decoupled by the Appelquist-Carazzone theorem and (2.12). In general strong quantum corrections might alter either of these properties. Thus in general we would need to add appropriate sources to the $\Lambda \rightarrow \infty$ action; compute the partition function by computing the $\Lambda \rightarrow 0$ limit of $\exp (-S)$; and finally explicitly test these properties by computing appropriate correlators (formed from differentiating with respect to the sources. This is the most general way to extract the results for physical quantities from $S$.) However since $g$ is perturbative at high energies (indeed $g \rightarrow 0$ as $\Lambda \rightarrow \infty$ ), we can be sure that the above deductions about the regulator fields, drawn at the perturbative level, are not destroyed by quantum corrections.

As already mentioned, we require that an ultraviolet regularisation at $\Lambda$, is implemented so that the right hand side of the flow equation makes sense. Note that this ensures that all further quantum corrections to $S$ (computed by solving for the flow at scales less than $\Lambda$ ) are cut off (smoothly) at $\Lambda$. Since momentum modes $p>\Lambda$ were fully contributing to the initial $\Lambda \rightarrow \infty$ partition function, and since $\mathcal{Z}$ is invariant under the flow, we can be sure that their effect has been incorporated $S$. In other words we can be sure that our final requirement on the flow, namely that it corresponds to integrating out momentum modes, has been incorporated.

(In refs. [9, 10], a possible further requirement on the flow equation, called "ultralocality" was discussed, replacing the usual notion of locality, although it was not clear that it was necessary however. We have seen here that the usual concept of locality is recovered providing the existence of a derivative expansion, invariance of $\mathcal{Z}$, and self-similar flow, are implemented. Furthermore the successful calculations of ref. [18] and here, confirm that the restriction of 'ultralocality' is unnecessary since they do not assume it.)

\subsection{Supergauge invariance and functional derivatives}

The peculiarities of $S U(N \mid N)$ affect functional derivatives with respect to $\mathcal{A}$ and lead to some constraints on the form of the exact RG if the flow equation is to be invariant under supergauge transformations.

As before [11, 16], it is convenient to define the functional derivatives of $\mathcal{C}$ and $\mathcal{A}$ so

as to extract the dual from under the supertrace. For an unconstrained field such as $\mathcal{C}$ we simply have 11, 16]:

$$
\frac{\delta}{\delta \mathcal{C}}:=\left(\begin{array}{cc}
\delta / \delta C^{1} & -\delta / \delta \bar{D} \\
\delta / \delta D & -\delta / \delta C^{2}
\end{array}\right)
$$

or in components

$$
\frac{\delta}{\delta \mathcal{C}}_{j}^{i}:={\frac{\delta}{\delta \mathcal{C}^{k}}}_{i} \sigma_{j}^{k}
$$


Under supergauge transformations (2.10), the functional derivative transforms as one would hope:

$$
\delta\left(\frac{\delta}{\delta \mathcal{C}}\right)=-i\left[\frac{\delta}{\delta \mathcal{C}}, \Omega\right]
$$

Such a derivative ${ }^{9}$ has the properties of 'supersowing' [11]:

$$
\frac{\partial}{\partial \mathcal{C}} \operatorname{str} \mathcal{C} Y=Y \quad \Longrightarrow \quad \operatorname{str} X \frac{\partial}{\partial \mathcal{C}} \operatorname{str} \mathcal{C} Y=\operatorname{str} X Y
$$

and 'supersplitting' [11]:

$$
\operatorname{str} \frac{\partial}{\partial \mathcal{C}} X \mathcal{C} Y=\operatorname{str} X \operatorname{str} Y
$$

i.e. of sowing two supertraces together, and splitting one supertrace into two, where $X$ and $Y$ are arbitrary constant supermatrices.

(N.B. as we will see later, it is a helpful trick to contract in arbitrary supermatrices at intermediate stages of the calculation: it allows manifestly $S U(N \mid N)$ invariant index-free calculations in the $S U(N \mid N)$ algebra, by permuting overall bosonic structures past each other. It also leads as we will show, to efficient diagrammatic techniques. The arbitrary supermatrices can always be stripped off at the end, if necessary. If we did not use this trick, we would lose manifest $S U(N \mid N)$ invariance at intermediate stages, by having to carry intermediate minus signs from fermionic parts of supermatrices anticommuted through each other.)

Since $\mathcal{A}$ is constrained to be supertraceless, its dual under the supertrace $\operatorname{str} \mathcal{J}_{\mu} \mathcal{A}_{\mu}$ has without loss of generality no 11 component: only

$$
\mathcal{J}_{\mu}-\frac{1}{2 N} \operatorname{tr} \mathcal{J}_{\mu}
$$

really couples. The natural construction for the $\mathcal{A}$ functional derivative from (2.3) 116]:

$$
\frac{\delta}{\delta \mathcal{A}_{\mu}}:=2 T_{A} \frac{\delta}{\delta \mathcal{A}_{A \mu}}+\frac{\sigma}{2 N} \frac{\delta}{\delta \mathcal{A}_{\mu}^{0}}
$$

pulls out precisely this combination. However from (2.6) and the completeness relations for the $T_{A}$ [16], under supergauge transformations

$$
\begin{aligned}
\delta\left(\frac{\delta}{\delta \mathcal{A}_{\mu}}\right) & =-i\left[\frac{\delta}{\delta \mathcal{A}_{\mu}}, \Omega\right]+\frac{i \mathbb{1}}{2 N} \operatorname{tr}\left[\frac{\delta}{\delta \mathcal{A}_{\mu}}, \Omega\right] \\
& =-i\left[\frac{\delta}{\delta \mathcal{A}_{\mu}}, \Omega\right]^{*} .
\end{aligned}
$$

The correction is to be expected since it ensures that $\delta / \delta \mathcal{A}$ remains traceless, but the fact that $\delta / \delta \mathcal{A}$ does not transform homogeneously means that supergauge invariance is destroyed unless $\delta / \delta \mathcal{A}$ is contracted under the supertrace into something that is supertraceless (causing the correction term to vanish). This is an extra constraint on the form of the flow equation.

\footnotetext{
${ }^{9}$ for simplicity, written with partial derivatives, to neglect the irrelevant spatial dependence
} 
[As an alternative one might try defining $\delta / \delta \mathcal{A}$ as only the $2 T_{A} \delta / \delta \mathcal{A}_{A}$ term in (2.20), however one can show from (2.6) that this does not transform into itself but into the full functional derivative given in $(2.20)$. It works however in the Bars* representation, where the transformation again takes the form (2.21).]

Similarly there are corrections to $(2.17)$ and $(2.18)$ that arise because the derivative is constrained: ${ }^{10}$

$$
\operatorname{str} X \frac{\partial}{\partial \mathcal{A}} \operatorname{str} \mathcal{A} Y=\operatorname{str} X Y-\frac{1}{2 N} \operatorname{str} X \operatorname{tr} Y
$$

as expected from (2.19), and

$$
\operatorname{str} \frac{\partial}{\partial \mathcal{A}} X \mathcal{A} Y=\operatorname{str} X \operatorname{str} Y-\frac{1}{2 N} \operatorname{tr} Y X .
$$

Since these corrections contain $\operatorname{tr} \cdots \equiv \operatorname{str} \sigma \cdots$, they similarly violate $S U(N \mid N)$ invariance. As we discuss in sec. 3.1, they also effectively disappear with the above constraint that $\delta / \delta \mathcal{A}$ is contracted into something supertraceless. (This is obvious in $(2.22)$ where thus $\operatorname{str} X=0$.)

In this way the supersplitting and supersowing rules actually become exact for both fields, even at finite $N$ (compare [10, 11]). As we will see, this leads to a very efficient diagrammatic technique incorporated into the Feynman diagrams, for evaluating the gauge algebra, analogous to the 't Hooft double line notation [29] and utilised earlier [9, 10, 11], but here applying even at finite $N$.

\subsection{Covariantisation}

Given some momentum space kernel $W_{p} \equiv W(p, \Lambda)$, we write in position space

$$
W_{x y} \equiv \int \frac{d^{4} p}{(2 \pi)^{4}} W(p, \Lambda) \mathrm{e}^{i p \cdot(x-y)},
$$

and introduce the shorthand:

$$
f \cdot W \cdot g:=\iint d^{4} x d^{4} y f(x) W_{x y} g(y),
$$

where $f$ and $g$ are any two functions. We define a general covariantisation of any such kernel (the 'wine' [10, 11]) via the supergauge invariant:

$$
\begin{aligned}
& u\{W\}_{\mathcal{A}} v= \\
& \sum_{m, n=0}^{\infty} \int d^{4} x d^{4} y d^{4} x_{1} \cdots d^{4} x_{n} d^{4} y_{1} \cdots d^{4} y_{m} W_{\mu_{1} \cdots \mu_{n}, \nu_{1} \cdots \nu_{m}}\left(x_{1}, \cdots, x_{n} ; y_{1}, \cdots, y_{m} ; x, y\right) \\
& \operatorname{str}\left[u(x) \mathcal{A}_{\mu_{1}}\left(x_{1}\right) \cdots \mathcal{A}_{\mu_{n}}\left(x_{n}\right) v(y) \mathcal{A}_{\nu_{1}}\left(y_{1}\right) \cdots \mathcal{A}_{\nu_{m}}\left(y_{m}\right)\right],
\end{aligned}
$$

where $u$ and $v$ are any two supermatrix representations, and where without loss of generality we may insist that $\{W\}_{\mathcal{A}}$ satisfies $u\{W\}_{\mathcal{A}} v \equiv v\{W\}_{\mathcal{A}} u$. We write the $m=0$ vertices (where there is no second product of gauge fields), more compactly as

$$
W_{\mu_{1} \cdots \mu_{n}}\left(x_{1}, \cdots, x_{n} ; x, y\right) \equiv W_{\mu_{1} \cdots \mu_{n},}\left(x_{1}, \cdots, x_{n} ; ; x, y\right),
$$

\footnotetext{
${ }^{10}$ ignoring the spacetime index and spatial dependence
} 
whilst the $m=n=0$ term is just the original kernel (2.24), i.e.

$$
W,(; ; x, y) \equiv W_{x y}
$$

We leave the covariantisation general, up to certain restrictions. One of these is already encoded into eq. (2.26), namely that there is just a single supertrace in (2.26), involving just two ordered products of supergauge fields. Another is that we require that the covariantisation satisfy coincident line identities [10] which in particular imply that if $v(y)=\mathbb{1} g(y)$ for all $y$, i.e. is in the scalar representation of the gauge group, then the covariantisation collapses to

$$
u\{W\}_{\mathcal{A}} v=(\operatorname{str} u) \cdot W \cdot g .
$$

As shown in ref. [11] ( $c f$. sec. 5.2 of that paper), the coincident line identities are equivalent to the requirement that the gauge fields in (2.26) all act by commutation. This requirement is necessary to ensure no- $\mathcal{A}^{0}$ remains valid and to ensure that $\delta / \delta \mathcal{A}$ is indeed contracted into something supertraceless. It is this that we need rather than the identities themselves, which are used just once, to collect terms in the calculation.

Although we will not use it explicitly, let us remark that these constraints are solved by the following general covariantisation [10, 11]:

$$
u\{W\}_{\mathcal{A}} v=\iint d^{4} x d^{4} y \int \mathcal{D}_{W} \ell_{x y} \operatorname{str} u(x) \Phi\left[\ell_{x y}\right] v(y) \Phi^{-1}\left[\ell_{x y}\right]
$$

where

$$
\Phi\left[\ell_{x y}\right]=P \exp -i \int_{\ell_{x y}} d z^{\mu} \mathcal{A}_{\mu}(z)
$$

is a path ordered exponential integral, i.e. a Wilson line, and the appearance of $\Phi^{-1}\left[\ell_{x y}\right]$ means that we traverse backwards along another coincident Wilson line. The covariantisation is determined by the measure $\mathcal{D}_{W}$ over configurations of the curves $\ell_{x y}$ and is so far left unspecified except for its normalisation:

$$
\int \mathcal{D}_{W} \ell_{x y} 1=W_{x y}
$$

as follows from (2.26) and (2.28). It is easy to see that 2.30) indeed does satisfy (2.29).

The expansion (2.26) can be represented as in fig. 1. As we explain later, these will act

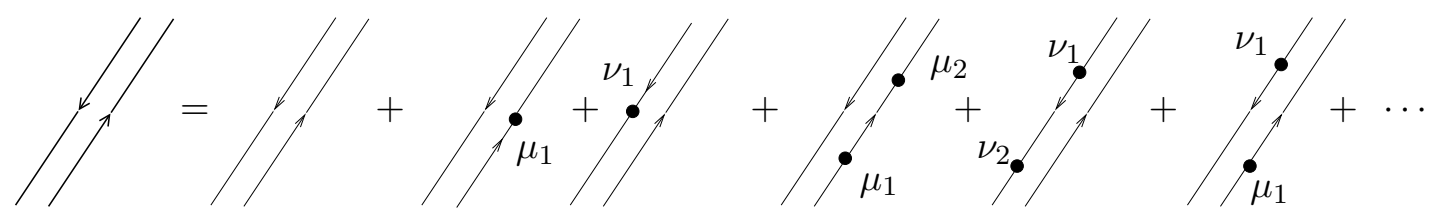

Figure 1: Wine expansion. The thick lines are expanded into thin lines, with the blobs representing $\mathcal{A}$ fields

as Feynman rules, although they can also be viewed directly as expansions of the Wilson lines (2.31) in the covariantised kernel (2.30). 
Finally, we will require that the covariantisation satisfies

$$
\frac{\delta}{\delta \mathcal{A}_{\mu}}\{W\}_{\mathcal{A}}=0
$$

(where the functional derivative acts on all terms inside $\{W\}_{\mathcal{A}}$ but not on the unspecified right hand attachment) i.e. that there are no diagrams in which the wine bites its own tail [9, 10, 11]. This leads to identities for the $W$ vertices which again we do not need in practice: as we will confirm, such terms do not in any case contribute to the one-loop $\beta$ function. However such diagrams do appear in general to lead to some improperly regularised terms and so some restriction is needed for consistency. We can use the representation (2.30) to see that sensible solutions to (2.33) do exist. For example we can simply insist that $\ell_{x y}$ is a straight Wilson line, and more generally that the measure $\mathcal{D}_{W}$ has no support on curves $\ell_{x y}$ that cross the points $x$ or $y$. The end points need defining carefully so that they only touch $x$ and $y$ after a limit has been taken [9]. However since we never specify the covariantisation, we only need to assume that solutions to (2.33) exist. In the calculation we just use (2.33) and thus just forbid all wine-biting-their-tail diagrams.

\subsection{Decoration with $\mathcal{C}$}

It will prove convenient to allow occurrences of $\mathcal{C}$ also on the Wilson lines (with the obvious corresponding extension of fig. (1) although we can limit their appearance to attachments at either end of $\ell_{x y}$. In this paper they will furthermore act only via commutation at both ends. Precisely, we extend the definition (2.26) so that

$$
u\{W\} v=u\{W\}_{\mathcal{A}} v-\frac{1}{4} \mathcal{C} \cdot u\left\{W_{m}\right\}_{\mathcal{A}} \mathcal{C} \cdot v,
$$

where $W_{m}(p, \Lambda)$ is some new kernel. In the expansion we now have vertices that come from both $\mathcal{A}$ and $\mathcal{C}$. Typically in this case $u$ and $v$ will actually correspond to functional differentials, with respect to, say, $Z_{1}$ and $Z_{2}$, and it will also be helpful to keep track of these flavours by including them as labels in the naming convention for the kernel, viz. as $W_{(m)}^{Z_{1} Z_{2}}$. The notation we will thus use in general is

$$
\begin{aligned}
& \frac{\delta}{\delta Z_{1}^{c}}\left\{W^{Z_{1} Z_{2}}\right\} \frac{\delta}{\delta Z_{2}^{c}}= \\
& \sum_{m, n=0}^{\infty} \int d^{4} x d^{4} y d^{4} x_{1} \cdots d^{4} x_{n} d^{4} y_{1} \cdots d^{4} y_{m} W_{\substack{a_{1} \cdots a_{n}, b_{1} \cdots b_{m} \\
X_{1} \cdots X_{n}, Y_{1} \cdots Y_{m}, Z_{1} Z_{2}}}\left(x_{1}, \cdots, x_{n} ; y_{1}, \cdots, y_{m} ; x, y\right) \\
& \operatorname{str}\left[\frac{\delta}{\delta Z_{1}^{c}(x)} X_{1}^{a_{1}}\left(x_{1}\right) \cdots X_{n}^{a_{n}}\left(x_{n}\right) \frac{\delta}{\delta Z_{2}^{c}(y)} Y_{1}^{b_{1}}\left(y_{1}\right) \cdots Y_{m}^{b_{m}}\left(y_{m}\right)\right],
\end{aligned}
$$

where the superfields $X_{i}, Y_{i}$ and $Z_{i}$, are $\mathcal{A}$ or $\mathcal{C}$, and the indices $a_{i}=\mu_{i}, b_{i}=\nu_{i}$ and $c=\gamma$ in the case that the corresponding field is $\mathcal{A}$ and null if the field is $\mathcal{C}$. In fact, as a consequence of the restricted structure (2.34), the $X_{2}, \cdots, X_{n-1}$ and $Y_{2}, \cdots, Y_{m-1}$ must be $\mathcal{A s}$ if they appear at all.

We can still insist without loss of generality that $u\{W\} v \equiv v\{W\} u$, and use the shorthand (2.27), where now we keep track of flavour labels as in (2.35) however. It is still 
the case that with no fields on the 'wine', the original $W$ kernel is recovered as in (2.28). The commutator structure in (2.34) ensures that (2.29) holds for the full 'wine' also:

$$
v(y)=\mathbb{1} g(y) \quad \forall y \quad \Rightarrow \quad u\{W\} v=(\operatorname{str} u) \cdot W \cdot g .
$$

Finally, the $\mathcal{C}$ s as further 'decorations' of the covariantised kernels are required to partake in the restriction described below (2.33), so this equation extends to

$$
\frac{\delta}{\delta \mathcal{A}_{\mu}}\{W\}=\frac{\delta}{\delta \mathcal{C}}\{W\}=0
$$

(In fact by $X=11$ in (2.18), the contribution from differentiating the leftmost $\mathcal{C}$ vanishes in any case.)

\subsection{Superfield expansion}

The Wilsonian effective action $S$ (and the seed action $\hat{S}$ that we will also introduce), being supergauge invariant, has an expansion in supertraces and products of supertraces:

$$
\begin{aligned}
& S=\sum_{n=1}^{\infty} \frac{1}{s_{n}} \int d^{4} x_{1} \cdots d^{4} x_{n} S_{a_{1} \cdots a_{n}}^{X_{1} \cdots X_{n}}\left(x_{1}, \cdots, x_{n}\right) \operatorname{str} X_{1}^{a_{1}}\left(x_{1}\right) \cdots X_{n}^{a_{n}}\left(x_{n}\right) \\
& +\frac{1}{2 !} \sum_{m, n=1}^{\infty} \frac{1}{s_{n} s_{m}} \int d^{4} x_{1} \cdots d^{4} x_{n} d^{4} y_{1} \cdots d^{4} y_{m} S_{a_{1} \cdots a_{n}, b_{1} \cdots b_{m}}^{X_{1} \cdots X_{n}, Y_{m}}\left(x_{1}, \cdots, x_{n} ; y_{1}, \cdots, y_{m}\right) \\
& \\
& +\operatorname{str} X_{1}^{a_{1}}\left(x_{1}\right) \cdots X_{n}^{a_{n}}\left(x_{n}\right) \operatorname{str} Y_{1}^{b_{1}}\left(y_{1}\right) \cdots Y_{m}^{b_{m}}\left(y_{m}\right) \\
& +\cdots,
\end{aligned}
$$

where again the $X_{i}^{a_{i}}$ are $\mathcal{A}_{\mu_{i}}$ or $\mathcal{C}$, and $Y_{j}^{b_{j}}$ are $\mathcal{A}_{\nu_{j}}$ or $\mathcal{C}$. (Note that throughout this paper we discard the vacuum energy.) Only one cyclic ordering of each list $X_{1} \cdots X_{n}$, $Y_{1} \cdots Y_{m}$ appears in the sum. Furthermore, if either list is invariant under some nontrivial cyclic permutations, then $s_{n}\left(s_{m}\right)$ is the order of the cyclic subgroup, otherwise $s_{n}=1$ $\left(s_{m}=1\right)$. (For example, in the terms where every $X_{i}^{a_{i}}$ is a $\mathcal{C}, s_{n}=n$.) The expansion can be represented diagrammatically, where a thick closed line stands for a single supertrace of any number of fields, as in fig. 2, and each blob represents a field in the supertrace, as in

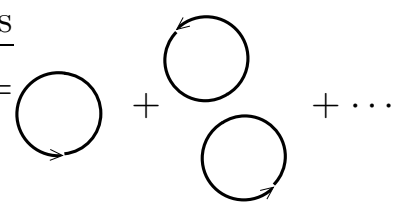

Figure 2: Expansion of the action in products of supertraces

fig. 3. In a somewhat similar way to (2.30) and (2.34), these closed lines can be interpreted as decorated Wilson loops [10, 11]. 


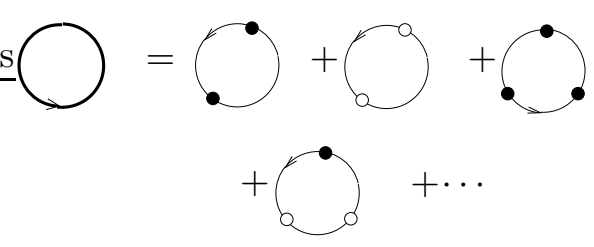

Figure 3: Expansion of a supertrace in powers of the fields $\mathcal{A}$ and $\mathcal{C}$.

When we spontaneously break the fermionic invariance by shifting $\mathcal{C}$ in the $\sigma$ direction, it will prove to be better to work separately with the bosonic and fermionic parts of the superfields. Thus we write in the broken phase

$$
\mathcal{A}_{\mu}=A_{\mu}+B_{\mu}, \quad \text { and } \quad \mathcal{C} \mapsto \mathcal{C}+\sigma=C+D+\sigma
$$

where $A$ and $C$ are the block diagonals, and $B$ and $D$ are the block off-diagonals in eqs. (2.1) and (2.9) respectively [11]. (We will see in the sec. 3 that $\mathcal{C}$ 's effective vacuum expectation value is just $\sigma$.)

Thus in the broken phase we will expand as in (2.38), but the flavours $X$ and $Y$ are set to $A, B, C$ or $D$. There will also be occurrences of $\sigma$. However since $\sigma$ commutes with $A$ and $C$, and anticommutes with $B$ and $D$, to define the expansion we can take the convention that we (anti)commute all such occurrences to the far right in the supertrace. Upon using $\sigma^{2}=\mathbb{1}$, we are then left with terms with either one $\sigma$ at the end of a supertrace or none at all in that supertrace. Since $\sigma$ has no position dependence, we put the flavour label in the superscript, but we omit the corresponding position label (and the term $n$ point vertex counts $n$ fields excluding $\sigma$ appearances). Clearly, since the broken fields can still be cyclically permuted by (anti)commutation through $\sigma$, we also omit it from the determination of the symmetry factor, i.e. $s_{n}$ is equal to the order of the cyclic permutation subgroup of the fields $X_{i}$, ignoring the $\sigma$ (if present). Finally note that each supertrace term must separately hold only totally bosonic combinations since if $X_{1} \cdots X_{n}$ (or $X_{1} \cdots X_{n} \sigma$ ) is fermionic, it is block off-diagonal and has vanishing supertrace.

Similarly, in (2.35), in the broken phase, $X, Y$ and $Z$ will be $A, B, C$ or $D$. Note that $Z_{1}$ can be the opposite statistic partner from $Z_{2}$. Since it is a single supertrace, again each contribution in (2.35) is overall bosonic however. Single occurrences of $\sigma$ can also appear at the ends of the Wilson lines, after taking into account that these can also (anti)commute through the $Z$ functional derivatives.

Finally, the momentum space vertices are written as

$$
S_{a_{1} \cdots a_{n}}^{X_{1} \cdots X_{n}}\left(p_{1}, \cdots, p_{n}\right)(2 \pi)^{4} \delta\left(\sum_{i=1}^{n} p_{i}\right)=\int d^{4} x_{1} \cdots d^{4} x_{n} \mathrm{e}^{-i \sum_{i} x_{i} \cdot p_{i}} S_{a_{1} \cdots a_{n}}^{X_{1} \cdots X_{n}}\left(x_{1}, \cdots, x_{n}\right)
$$

where all momenta are taken pointing into the vertex, and similarly for all the other vertices including (2.35). We use the short hand $S_{a b}^{X Y}(p) \equiv S_{a b}^{X Y}(p,-p)$ and $S_{a b}^{X Y \sigma}(p) \equiv$ $S_{a b}^{X Y \sigma}(p,-p)$ for action two-point vertices. 


\section{A manifestly $S U(N \mid N)$ gauge invariant exact RG}

Our strategy is to write down a manifestly supergauge invariant flow equation, obeying the rules outlined in sec. 2, and then spontaneously break it. Defining $\Sigma_{g}=g^{2} S-2 \hat{S}$, we simply set

$$
\Lambda \partial_{\Lambda} S=-a_{0}\left[S, \Sigma_{g}\right]+a_{1}\left[\Sigma_{g}\right]
$$

where

$$
a_{0}\left[S, \Sigma_{g}\right]=\frac{1}{2} \frac{\delta S}{\delta \mathcal{A}_{\mu}}\left\{\dot{\Delta}^{\mathcal{A} \mathcal{A}}\right\} \frac{\delta \Sigma_{g}}{\delta \mathcal{A}_{\mu}}+\frac{1}{2} \frac{\delta S}{\delta \mathcal{C}}\left\{\dot{\Delta}^{\mathcal{C C}}\right\} \frac{\delta \Sigma_{g}}{\delta \mathcal{C}},
$$

and

$$
a_{1}\left[\Sigma_{g}\right]=\frac{1}{2} \frac{\delta}{\delta \mathcal{A}_{\mu}}\left\{\dot{\Delta}^{\mathcal{A A}}\right\} \frac{\delta \Sigma_{g}}{\delta \mathcal{A}_{\mu}}+\frac{1}{2} \frac{\delta}{\delta \mathcal{C}}\left\{\dot{\Delta}^{\mathcal{C C}}\right\} \frac{\delta \Sigma_{g}}{\delta \mathcal{C}} .
$$

In the rest of this section we explain the meaning of the various components, at the same time developing some of the properties of this exact RG.

The definition of $\Sigma_{g}$ and the form of the flow equation (3.1) are the same as in refs. [10, 11]. In contrast to ref. 11] however, the exact RG is very simple in conception. The basic structure is inherited from the Wilson exact RG [2, 30, 9]: the bilinear functional $-a_{0}$ generates the classical corrections, whilst the linear functional $a_{1}$ generates quantum corrections. As in refs. 10, 11], $a_{1}$ has exactly the same structure as $a_{0}$ except that the leftmost functional derivatives differentiate everything to their right. Consequently we have

$$
\Lambda \partial_{\Lambda} \mathrm{e}^{-S}=a_{1}\left[\Sigma_{g} \mathrm{e}^{-S}\right]
$$

which shows that condition (2.13) is fulfilled.

As before, $g(\Lambda)$ is the renormalized coupling of the $S U(N)$ Yang-Mills theory carried by $A^{1}$. It is defined through the renormalization condition:

$$
S\left[\mathcal{A}=A^{1}, \mathcal{C}=\overline{\mathcal{C}}\right]=\frac{1}{2 g^{2}} \operatorname{tr} \int d^{4} x\left(F_{\mu \nu}^{1}\right)^{2}+\cdots,
$$

where the ellipsis stands for higher dimension operators (and the ignored vacuum energy),

and $\overline{\mathcal{C}}$ is the effective vacuum expectation value defined so as to minimise the effective potential $V(\mathcal{C})$ in $S$ :

$$
\left.\frac{\partial V}{\partial \mathcal{C}}\right|_{\mathcal{C}=\overline{\mathcal{C}}}=0
$$

$\overline{\mathcal{C}}$ is spacetime independent and generically contains terms proportional to $\sigma$ and 11 [16]. We will see later that for the purposes of this paper we can simply set $\overline{\mathcal{C}}=\sigma$.

The similarities mean that the general structure of the perturbative expansion is the same as in refs. [10, 11]: we see from (3.1), that $S \sim 1 / g^{2}$ at the classical level [consistent with (3.5)], and by iteration, using (3.1), that $S$ has as expected the weak coupling expansion

$$
S=\frac{1}{g^{2}} S_{0}+S_{1}+g^{2} S_{2}+\cdots
$$


where $S_{0}$ is the classical effective action, $S_{1}$ the one-loop correction, and so on. Substituting this expansion in (3.1), we see that the $\beta$ function must also take the standard form

$$
\beta:=\Lambda \frac{\partial g}{\partial \Lambda}=\beta_{1} g^{3}+\beta_{2} g^{5}+\cdots
$$

with coefficients to be determined. From (3.7) and (3.8), we obtain the loopwise expansion of (3.1):

$$
\begin{aligned}
\Lambda \frac{\partial}{\partial \Lambda} S_{0} & =-a_{0}\left[S_{0}, S_{0}-2 \hat{S}\right] \\
\Lambda \frac{\partial}{\partial \Lambda} S_{1} & =2 \beta_{1} S_{0}-2 a_{0}\left[S_{0}-\hat{S}, S_{1}\right]+a_{1}\left[S_{0}-2 \hat{S}\right]
\end{aligned}
$$

etc. Actually, we will find it convenient to add some simple quantum corrections to the supergauge invariant seed action $\hat{S}$, giving it a $g$ dependence (as we outline below). We also need to take account of the flow of $g_{2}$, the coupling for the second $S U(N)$ carried by $A_{2}$. However, neither of these complications has an effect on the one-loop $\beta$ function computation, so will be largely ignored here, and developed fully only when we consider multi-loops [1].

$\hat{S}$ is used to determine the form of the classical effective kinetic terms and the kernels $\dot{\Delta}(p, \Lambda)$. It therefore has to incorporate the covariant higher derivative regularisation and allow the spontaneous symmetry breaking we require. Unlike previously [9, 10, 11], we will see that we otherwise leave it almost entirely unspecified. The kernels $\dot{\Delta}$ are determined by the requirement that after spontaneous symmetry breaking, the two-point vertices of the classical effective action $S_{0}$ and $\hat{S}$ can be set equal. As previously [9, 10, 11], this is imposed as a useful technical device, since it allows classical vertices to be immediately solved in terms of already known quantities. It also means that the integral of the kernels defined via

$$
\Lambda \partial_{\Lambda} \Delta=-\dot{\Delta}
$$

will play a closely similar rôle to that of propagators, in particular being the inverse of these two-point vertices up to gauge transformations.

The $\mathcal{C}$ commutator terms in (2.34), yield $\sigma$ commutators on spontaneous symmetry breaking. Since $\sigma$ commutes with $A$ and $C$ but anticommutes with $B$ and $D, \Delta_{m}^{\mathcal{A A}}$ and $\Delta_{m}^{\mathcal{C C}}$ allow for the addition of spontaneous mass creation for $B$ and $D$ whilst still allowing the solution that the two-point vertices of $\hat{S}$ and $S_{0}$ are equal. The appearance of the $\mathcal{C}$ commutator on both sides allows us to insist that $\mathcal{C} \leftrightarrow-\mathcal{C}$ is an invariance of the symmetric phase. The form (3.2, 3.3) preserves charge conjugation symmetry $\mathcal{C} \mapsto \mathcal{C}^{T}$, $\mathcal{A} \mapsto-\mathcal{A}^{T}$ (using the definition of the supermatrix transpose in ref. [16]. Note that here the transformation for $\mathcal{C}$ is as given so that its vacuum expectation value is invariant under charge conjugation. Since charge conjugation reverses the order of terms in a supertrace, diagrammatically it corresponds to reversing the arrows in figs. 1 - 3, i.e. to mirror reflection [10, 11].) 
From 2.16) and sec. 2.5, it is trivial to see that the $\delta / \delta \mathcal{C}$ terms are supergauge invariant. Under a supergauge transformation we have by (2.21) and (2.36),

$$
\delta\left(\frac{\delta S}{\delta \mathcal{A}_{\mu}}\left\{\dot{\Delta}^{\mathcal{A} \mathcal{A}}\right\} \frac{\delta \Sigma_{g}}{\delta \mathcal{A}_{\mu}}\right)=\frac{i}{2 N} \operatorname{tr}\left[\frac{\delta S}{\delta \mathcal{A}_{\mu}}, \Omega\right] \cdot \dot{\Delta}^{\mathcal{A A}} \cdot \operatorname{str} \frac{\delta \Sigma_{g}}{\delta \mathcal{A}_{\mu}}+\left(S \leftrightarrow \Sigma_{g}\right),
$$

where $S \leftrightarrow \Sigma_{g}$ stands for the same term with $S$ and $\Sigma_{g}$ interchanged. But by (2.20) and no- $\mathcal{A}^{0}$

$$
\operatorname{str} \frac{\delta \Sigma_{g}}{\delta \mathcal{A}_{\mu}}=\frac{\delta \Sigma_{g}}{\delta \mathcal{A}_{\mu}^{0}}=0
$$

similarly for $S$, and thus the tree-level terms are supergauge invariant. Similarly, the quantum terms are $S U(N \mid N)$ gauge invariant, since

$$
\delta\left(\frac{\delta}{\delta \mathcal{A}_{\mu}}\left\{\dot{\Delta}^{\mathcal{A} \mathcal{A}}\right\} \frac{\delta}{\delta \mathcal{A}_{\mu}} \Sigma_{g}\right)=\frac{i}{N} \operatorname{tr}\left[\frac{\delta}{\delta \mathcal{A}_{\mu}}, \Omega\right] \cdot \dot{\Delta}^{\mathcal{A} \mathcal{A}} \cdot \operatorname{str} \frac{\delta \Sigma_{g}}{\delta \mathcal{A}_{\mu}}=0 .
$$

This completes the proof that the exact RG is supergauge invariant!

Note that there is no point in incorporating longitudinal terms into the exact RG (as was done in ref. [11]) because here the manifest supergauge invariance means that they can be exchanged for $\mathcal{C}$ commutators:

$$
\nabla_{\mu} \cdot \frac{\delta S}{\delta \mathcal{A}_{\mu}}=i \mathcal{C} \cdot \frac{\delta S}{\delta \mathcal{C}}
$$

(as holds for any supergauge invariant functional) and thus absorbed into the $\dot{\Delta}_{m}^{\mathcal{C C}}$ term.

It is important for the working of the $S U(N \mid N)$ regularisation that the effective scale of spontaneous symmetry breaking is tied to the higher derivative regularisation scale, which thus both flow with $\Lambda$. This is not the typical situation, but can be arranged to happen here by constraining $\hat{S}$ appropriately. However, as we now show, the constraint is straightforward only if we take $\mathcal{C}$ to be dimensionless in (3.1) - (3.3).

Contracting an arbitrary constant supermatrix $X$ into (3.6) (for convenience, $c f$. sec. 2.3) and differentiating with respect to $\Lambda$, we have:

$$
\left[\operatorname{str} \frac{\partial \overline{\mathcal{C}}}{\partial \Lambda} \frac{\partial}{\partial \mathcal{C}} \operatorname{str} X \frac{\partial V}{\partial \mathcal{C}}+\operatorname{str} X \frac{\partial}{\partial \mathcal{C}} \frac{\partial V}{\partial \Lambda}\right]_{\mathcal{C}=\overline{\mathcal{C}}}=0 .
$$

We can compute the flow $\partial V / \partial \Lambda$ by setting $\mathcal{A}=0$ and $\mathcal{C}=\overline{\mathcal{C}}$ in (3.1). Taking the classical limit $V \rightarrow V_{0}$, we find that the resulting equation simplifies dramatically. Using eqns. (3.9), (3.2), (3.6), (2.26), (2.28), the fact that vertices in the actions with only one $\mathcal{A}_{\mu}$, vanish at zero momentum (by Lorentz invariance), and

$$
\left.\left[\overline{\mathcal{C}}, \frac{\partial \hat{V}}{\partial \mathcal{C}}\right]\right|_{\mathcal{C}=\overline{\mathcal{C}}}=0
$$

which follows from global $S U(N \mid N)$ invariance (where $\hat{V}$ is the potential in $\hat{S}$ ), we get

$$
\operatorname{str}\left[\left(\Lambda \frac{\partial \overline{\mathcal{C}}}{\partial \Lambda}+\dot{\Delta}^{\mathcal{C C}}(0, \Lambda) \frac{\partial \hat{V}}{\partial \mathcal{C}}\right) \frac{\partial}{\partial \mathcal{C}} \operatorname{str} X \frac{\partial V_{0}}{\partial \mathcal{C}}\right]_{\mathcal{C}=\overline{\mathcal{C}}}=0
$$

With $\mathcal{C}$ dimensionless, we can and will insist that the classical vacuum expectation value $\overline{\mathcal{C}}=\sigma$. Eq. (3.18) is then satisfied if and only if ${ }^{11} \hat{V}$ also has a minimum at $\mathcal{C}=\sigma$.

${ }^{11}$ We will see that the requirement that $C$ has a mass in the broken phase forces $\dot{\Delta}^{\mathcal{C C}}(0, \Lambda) \neq 0$. 
This is delightful since it ensures that at the classical level at least, neither action has one-point $\mathcal{C}$ vertices in the broken phase. We will thus impose

$$
\left.\frac{\partial \hat{V}}{\partial \mathcal{C}}\right|_{\mathcal{C}=\sigma}=0
$$

as a constraint on $\hat{S}$.

Had we not taken $\mathcal{C}$ to be dimensionless, we would have had to require that $\overline{\mathcal{C}}$ depend on $\Lambda$, in order that the effective breaking scale flows with $\Lambda$. Since $X$ is general, (3.18) would then imply that $\hat{V}$ cannot have a minimum also at $\mathcal{C}=\overline{\mathcal{C}}$. Further analysis shows that $\hat{V}$ is then forced to violate $\mathcal{C} \leftrightarrow-\mathcal{C}$ symmetry in the symmetric phase.

Although conventionally $\mathcal{C}$ would have dimension one, for these reasons we will take it to be dimensionless from now on. (It is intriguing that the conclusion that $\mathcal{C}$ [actually $C$ ] must be dimensionless was reached for very different reasons in refs. [9, 11] which are no longer necessarily applicable, now that (3.15) is a symmetry.)

At the quantum level, $\overline{\mathcal{C}}=\sigma$ can be expected to receive loop corrections. Since $S U(N) \times S U(N)$ invariance is left unbroken, these corrections can only be proportional to $\sigma$ or 1 . Corrections proportional to the latter do not affect the breaking (but presumably through (3.5) give important contributions at higher loops), however corrections proportional to $\sigma$ would result, through (3.15), in broken gauge invariance identities that explicitly involve $g$ and thus mix different loop orders. We can avoid this by again using the freedom in our choice of $\hat{S}$ to design things appropriately. We can constrain the appearance of $\hat{V}$ one-point vertices in the broken phase

$$
v^{C} \operatorname{str} \mathcal{C}+v^{C \sigma} \operatorname{str} \mathcal{C} \sigma
$$

by imposing $\overline{\mathcal{C}}=\sigma$ as a renormalization condition. Each $v$ is then a non-vanishing function of $g$, but from the analysis above, only from one-loop onwards:

$$
v^{C}(g)=v_{1}^{C} g^{2}+v_{2}^{C} g^{4}+\cdots \quad \text { and } \quad v^{C \sigma}(g)=v_{1}^{C \sigma} g^{2}+v_{2}^{C \sigma} g^{4}+\cdots .
$$

However the fact that these corrections start only at one loop, makes them already too high an order to affect the one-loop $\beta$ function calculation. (This is particularly clear from the perspective of higher loop calculations [1].)

\subsection{Supersowing and supersplitting in the $\mathcal{A}$ sector}

The inherent supersymmetry has a remarkable effect on the gauge algebra: one can replace the usual manipulation of structure constants and reduction to Casimirs, which becomes increasingly involved at higher loops, by simple steps (2.17) and (2.18) which always either just sow together supertraces or split them open. These have an immediate diagrammatic interpretation. The apparent violations present in (2.22) and (2.23) must somehow disappear since they would violate even global $S U(N \mid N)$. We first prove that this indeed the case.

For the case where the action contains just a single supertrace, which will turn out to be all we need here, we could adapt the proof given in sec. 6.2 of ref. [16]. However, in 
preparation for future work, we will give a more sophisticated proof which is applicable when working with multiple supertrace contributions. Indeed we will see that there is then one special case, where the corrections in (2.22,2.23) do survive, and result in a simple supergauge invariant correction.

The corrections present in $2.22,2.23)$ arise because $\mathcal{A}$ is constrained to be supertraceless. To compare their effect to the unconstrained case (2.17,2.18), we momentarily 'lift' $\mathcal{A}$ to a full superfield $\mathcal{A}^{e}$ by adding a $\sigma$ part:

$$
\mathcal{A}_{\mu} \mapsto \mathcal{A}_{\mu}^{e}:=\mathcal{A}_{\mu}+\sigma \mathcal{A}_{\mu}^{\sigma}
$$

$\mathcal{A}_{\mu}^{\sigma}$ is taken arbitrary so the map is not at all unique. We similarly extend all functionals of $\mathcal{A}$ to the full space, simply by replacing $\mathcal{A}$ with $\mathcal{A}^{e}$, e.g.

$$
S^{e}\left[\mathcal{A}^{e}, \mathcal{C}\right]:=S\left[\mathcal{A} \mapsto \mathcal{A}^{e}, \mathcal{C}\right]
$$

Again, this is a not unique procedure, as can be seen for example in the fact that $\operatorname{str} \mathcal{A}$ vanishes, but the promoted functional $\operatorname{str} \mathcal{A}^{e}$ does not. We also introduce the projection back onto the supertraceless space:

$$
\pi \mathcal{A}_{\mu}^{e}=\mathcal{A}_{\mu}, \quad \pi S^{e}=S, \quad \text { etc. },
$$

which of course is unique. Functional derivatives with respect to $\mathcal{A}^{e}$ can be written as

$$
\frac{\delta}{\delta \mathcal{A}_{\mu}^{e}}=\frac{\delta}{\delta \mathcal{A}_{\mu}}+\frac{1}{2 N} \frac{\delta}{\delta \mathcal{A}_{\mu}^{\sigma}}
$$

using $(2.20)$, or equivalently defined as in (2.15). $\delta / \delta \mathcal{A}^{e}$ thus satisfies the exact supersowing and supersplitting relations $2.17,2.18$ ). In the extended space, the constrained derivative 2.20) can now be written in terms of an unconstrained derivative:

$$
\frac{\delta}{\delta \mathcal{A}_{\mu}}=\frac{\delta}{\delta \mathcal{A}_{\mu}^{e}}-\frac{1}{2 N} \operatorname{tr} \frac{\delta}{\delta \mathcal{A}_{\mu}^{e}}
$$

Of course $\pi$ and $\delta / \delta \mathcal{A}^{\sigma}$ do not commute, however

$$
\frac{\delta S}{\delta \mathcal{A}_{\mu}}\left\{\dot{\Delta}^{\mathcal{A A}}\right\} \frac{\delta \Sigma_{g}}{\delta \mathcal{A}_{\mu}}=\pi\left\{\frac{\delta S^{e}}{\delta \mathcal{A}_{\mu}}\left\{\dot{\Delta}^{\mathcal{A A}}\right\}^{e} \frac{\delta \Sigma_{g}^{e}}{\delta \mathcal{A}_{\mu}}\right\}
$$

since $\mathcal{A}^{\sigma}$ is not differentiated on the right hand side. Substituting (3.26) or (3.25), and using (2.36) and (2.20), the term in big curly braces becomes

$$
\frac{\delta S^{e}}{\delta \mathcal{A}_{\mu}^{e}}\left\{\dot{\Delta}^{\mathcal{A} \mathcal{A}}\right\}^{e} \frac{\delta \Sigma_{g}^{e}}{\delta \mathcal{A}_{\mu}^{e}}-\frac{1}{2 N} \frac{\delta \mathcal{S}^{e}}{\delta \mathcal{A}_{\mu}^{0}} \cdot \dot{\Delta}^{\mathcal{A} \mathcal{A}} \cdot \frac{\delta \Sigma_{g}^{e}}{\delta \mathcal{A}_{\mu}^{\sigma}}-\frac{1}{2 N} \frac{\delta \Sigma_{g}^{e}}{\delta \mathcal{A}_{\mu}^{0}} \cdot \dot{\Delta}^{\mathcal{A A}} \cdot \frac{\delta \mathcal{S}^{e}}{\delta \mathcal{A}_{\mu}^{\sigma}} .
$$

Now, as we explain below, no- $\mathcal{A}^{0}$ symmetry is violated in the extended space. However the $\mathcal{A}^{0}$ derivatives in $(3.28)$ do vanish after the projection. Thus 3.27 becomes

$$
\frac{\delta S}{\delta \mathcal{A}_{\mu}}\left\{\dot{\Delta}^{\mathcal{A} \mathcal{A}}\right\} \frac{\delta \Sigma_{g}}{\delta \mathcal{A}_{\mu}}=\pi\left\{\frac{\delta S^{e}}{\delta \mathcal{A}_{\mu}^{e}}\left\{\dot{\Delta}^{\mathcal{A} \mathcal{A}}\right\}^{e} \frac{\delta \Sigma_{g}^{e}}{\delta \mathcal{A}_{\mu}^{e}}\right\},
$$


which says precisely that the corrections in (2.22) can be ignored: exactly the same result is obtained if exact supersowing is used.

However, performing the same analysis on the corresponding quantum term in (3.3), we get a correction to exact supersplitting, consisting of an attachment of the (zero-point) kernel $\dot{\Delta}^{\mathcal{A A}}(p, \Lambda)$ to two $\mathcal{A}$ points in $\Sigma_{g}$ :

$$
\left.\frac{\delta}{\delta \mathcal{A}_{\mu}}\left\{\dot{\Delta}^{\mathcal{A} \mathcal{A}}\right\} \frac{\delta \Sigma_{g}}{\delta \mathcal{A}_{\mu}}=\pi\left\{\frac{\delta}{\delta \mathcal{A}_{\mu}^{e}}\left\{\dot{\Delta}^{\mathcal{A} \mathcal{A}}\right\}\right\}^{e} \frac{\delta \Sigma_{g}^{e}}{\delta \mathcal{A}_{\mu}^{e}}\right\}-\frac{1}{N} \pi \frac{\delta}{\delta \mathcal{A}_{\mu}^{\sigma}} \cdot \dot{\Delta}^{\mathcal{A} \mathcal{A}} \cdot \frac{\delta \Sigma_{g}}{\delta \mathcal{A}_{\mu}^{0}} .
$$

To understand when this correction is non-vanishing, we need briefly to analyse the consequences of no- $\mathcal{A}^{0}$ symmetry in more detail. Considering the transformation ${ }^{12} \delta \mathcal{A}_{\mu}=\lambda_{\mu} 1$ in (2.38), we see that the result must vanish either via the supergroup algebra because the corresponding vertex contains a factor $\operatorname{str} \mathcal{A} \mathcal{A}$, thus generating $\operatorname{str} \mathcal{A}=0$ (but $\operatorname{str} \mathcal{A}^{e} \neq 0$ in the extended space), or because a non-trivial constraint exists on the corresponding vertex function. (This is simply that the sum over all possible valid placings of $\mathcal{A}^{0}$ s associated position and Lorentz argument inside a vertex function leaving other arguments alone, yields zero, $c f$. (3.5) and refs. [31, 1] for more detail.) This non-trivial constraint then causes the coefficient to vanish whether or not the remaining supergauge fields are extended by $\mathcal{A}^{\sigma} \sigma$. Thus the correction in (3.30) vanishes in all cases except where the zero-point $\dot{\Delta}^{\mathcal{A A}}$ kernel attaches each end to a $\operatorname{str} \mathcal{A} \mathcal{A}$ factor. Comparing the result to the computation assuming exact supersplitting, i.e. the first term in (3.30), we see that instead of getting a supergroup factor $(\operatorname{str} \mathbb{1})^{2}=0$ we get $-\frac{1}{N} \operatorname{str} \sigma$ i.e. a supergroup factor of -2 .

(Note that in deriving this rule we have assumed that vertices in $\Sigma_{g}$ with factors $\operatorname{str} \mathcal{A}$ have been set to zero from the beginning [as would follow immediately from the $S U(N \mid N)$ group theory]. If for some reason this was not done then the first term in (3.30) can get a non-zero contribution from the kernel attaching to this $\operatorname{str} \mathcal{A}=2 N \mathcal{A}^{\sigma}$ point. However it then also appears in the correction with precisely equal and opposite coefficient.)

This supergroup factor should have been expected since the algebra part of the attachment of a zero-point kernel to a two-point vertex simply counts the number of bosonic degrees of freedom in the algebra minus the number of fermionic degrees of freedom. There are $N^{2}$ fermionic such terms in $B$, but only $N^{2}-2$ in $A$, since both $\mathcal{A}^{\sigma}$ and, by no- $\mathcal{A}^{0}$ symmetry, $\mathcal{A}^{0}$, are missing.

Since the correction in (3.30) is non-vanishing only when using up a separate $\operatorname{str} \mathcal{A} \mathcal{A}$ factor, it is clear that the result is still supergauge invariant in the remaining external superfields. Furthermore in the present case where we will be able to work with actions with only a single supertrace, the entire effect of the correction is a just vacuum energy contribution, which from now on we ignore.

\subsection{Diagrammatic interpretation}

$\mathcal{A}$ thus also effectively satisfies the exact supersowing and supersplitting relations (2.17) and (2.18). By using these equations when the covariantised kernels (2.35) act on the

\footnotetext{
${ }^{12}$ there are higher order constraints from separating out higher powers of $\mathcal{A}^{0}$ but from (3.30) we only need the first order
} 
actions (2.38), and comparing the result to the diagrammatic interpretation of the covariantised kernels and actions, fig. 1 and figs. 2, 3, it is clear that the exact RG is given diagrammatically as in fig. 1 .

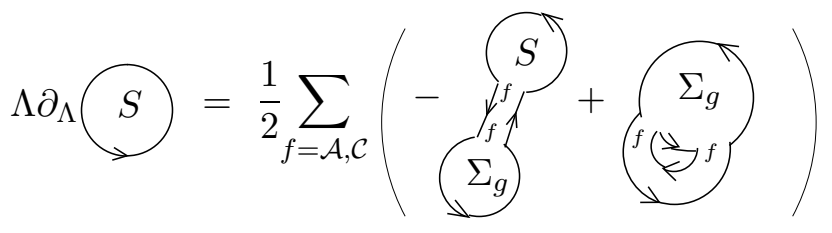

Figure 4: Graphical representation of the exact RG, when $S$ and $\hat{S}$ contain only single supertraces.

Here we have specialised to the case of interest in this paper, where $S$ and $\hat{S}$ can be assumed to have only a single supertrace. (The extension to the more general contributions fig. 2 is obvious.) Expanding the thick lines (representing any number of fields) into a power series in the fields, we translate the figure into individual Feynman diagrams, whose Feynman rules are given by the momentum space versions of the vertices in (2.35) and (2.38) (without the symmetry factors). ${ }^{13}$ The points representing individual fields and their associated momenta and Lorentz indices, appear in all places on a composite loop with equal weight, whilst respecting the cyclic order. Of course if one of the corresponding vertices does not appear in the expansions (2.35) and (2.38), the corresponding Feynman rule is zero.

It can be seen from fig. 团 that the tree-level corrections preserve the assumption that there is only a single supertrace in $S$, but that each quantum correction results in an extra supertrace factor. Thus in general $S$ has terms with any number of supertraces, and already a product of two supertraces at one-loop. However for the computation of the $\beta$ function, we need only look at contributions to the $A A$ two-point vertex (see (3.5) and later, or refs. [9, 10, 11]). Since $A$ is both traceless and supertraceless, to get a non-vanishing answer both $A$ s must lie in the same supertrace, leaving the other one empty of fields. In this way, $S$ effectively contains only a single supertrace to the order in which we are working.

\subsection{After spontaneous breaking}

We substitute $\mathcal{C} \mapsto \mathcal{C}+\sigma$, and from now on work in the spontaneously broken phase. Working with fields appropriate for the remaining $S U(N) \times S U(N)$ symmetry, we break $\mathcal{A}$ and $\mathcal{C}$ down to their bosonic and fermionic parts $A, B, C$ and $D$ as in (2.39).

The diagrammatic interpretation is still the same, except that we now have the four flavours to scatter around the composite loops, and appearances of $\sigma$, which can be simplified as explained in sec. 2.6. (Some terms are then related, for example $\dot{\Delta}_{\mu}^{A, A A}=\dot{\Delta}_{\mu}^{B, A B}$, although we never need to use this explicitly.) In addition, we must recall the corrections to supersplitting and supersowing arising from differentiating only partial supermatrices [11. These lead to further appearances of $\sigma$ which are easily computed by expressing the

\footnotetext{
${ }^{13}$ This part of the analysis is the same as in ref. [11, except that here we make explicit the factor of $1 / 2$ from (3.2) and (3.3), in fig. 1 and the Feynman diagrams, and the factor of $1 / \Lambda^{2}$ is now incorporated in the definition of the kernels $\dot{\Delta}$.
} 
partial supermatrices in terms of full supermatrices via the projectors $d_{ \pm}$onto the block (off)diagonal components

$$
\mathrm{d}_{ \pm} X=\frac{1}{2}(X \pm \sigma X \sigma),
$$

(hence $C=\mathrm{d}_{+} \mathcal{C}, D=\mathrm{d}_{-} \mathcal{C}$, etc. ). Diagrammatically this simply amounts to corrections involving a pair of $\sigma$ s inserted either side of the attachment as in fig. 司 [11].

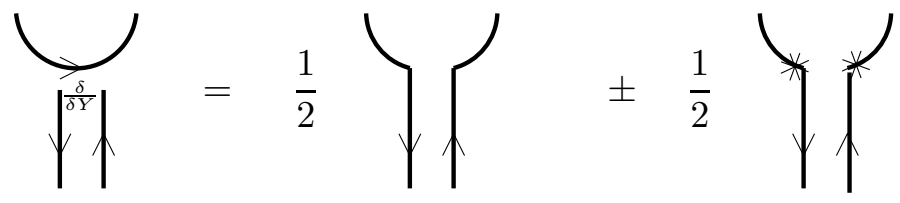

Figure 5: Feynman diagram representation of attachment via a partial supermatrix $\mathrm{d}_{ \pm} Y= \pm Y$.

For tree-level type attachments as in (2.17), the corrections merely ensure that the coefficient supermatrices $(X$ and $Y$ ) have the appropriate statistics to make each supertrace term totally bosonic ( $c f$. sec. 2.6), but this has already been taken into account in the Feynman rules. Thus these corrections have no effect at tree level [11].

Since the classical action $S_{0}$ (similarly $\hat{S}$ ) has only a single supertrace and respects $\mathcal{C} \leftrightarrow-\mathcal{C}$ invariance in the symmetric phase ( $c f$. sec. 3), upon spontaneous breaking we have the 'theory space' symmetry

$$
\begin{aligned}
C & \leftrightarrow-C, \\
D & \leftrightarrow-D, \\
\sigma & \leftrightarrow-\sigma .
\end{aligned}
$$

The single supertrace part of the one-loop effective action $S_{1}$ has a single supertrace because it also has a supertrace void of fields ( $c f$. sec. 3.2). In order for this not to vanish it must 'trap' a $\sigma$ (so that we get $\operatorname{str} \sigma=2 N$ rather than str $\mathbb{1}=0$ ). Therefore, the non-trivial supertrace has one less $\sigma(\bmod$ two) and is thus odd under the symmetry (3.32).

These observations, which can be easily extended to multiple loops and supertraces, are useful in limiting the possible vertices.

\section{4 (Un)broken gauge invariance}

Splitting $\Omega$ into its fermionic and bosonic parts: $\tau=\mathrm{d}_{-} \Omega$ and $\omega=\mathrm{d}_{+} \Omega$, we obtain from (2.4), (2.6) and (2.10) the unbroken $S U(N) \times S U(N)$ transformations

$$
\begin{aligned}
\delta A_{\mu} & =D_{\mu} \cdot \omega \\
\delta B_{\mu} & =-i B_{\mu} \cdot \omega \\
\delta C & =-i C \cdot \omega \\
\delta D & =-i D \cdot \omega,
\end{aligned}
$$

where $D_{\mu}=\partial_{\mu}-i A_{\mu}$ is the covariant derivative for the $S U(N) \times S U(N)$ (the dot again means action by commutation, and we have used the fact that $[\sigma, \omega]=0)$ and the broken 
fermionic gauge transformations

$$
\begin{aligned}
\delta B_{\mu} & =D_{\mu} \cdot \tau \\
\delta A_{\mu} & =-i B_{\mu} \cdot \tau \\
\delta D & =-i C \cdot \tau+2 i \tau \sigma \\
\delta C & =-i D \cdot \tau .
\end{aligned}
$$

From the first of (3.33) we see that $A$ can have no wavefunction renormalization because if it did, then replacing $A$ by $Z^{1 / 2} A$, this becomes $\delta A_{\mu}=D_{\mu} \cdot \omega+\left(Z^{-1 / 2}-1\right) \partial_{\mu} \omega$, i.e. the gauge symmetry is violated [9, 10, 11]. (Clearly this is true whether or not one tries to reparametrize $\omega$ also.) This is the reason for scaling the coupling $g$ out of the connection (2.4): $A$ then has no anomalous dimension and only $g$ renormalizes. Since the corresponding marginal term to (3.5) exists for $A_{2}$, there is also a coupling $g_{2}$. Its renormalization is important for calculations in the physical sector beyond one loop [1].

In a similar way the first two relations of (3.34) imply that $B$ can have no wavefunction renormalization, whilst at first surprisingly the last two relations imply that $C$ and $D$ cannot have any wavefunction renormalization either. However, these last two relations are a consequence of the vacuum expectation value $\overline{\mathcal{C}}=\sigma$ being protected by the introduction of the terms $3.20,3.21$.

Applying these relations to the field expansions in momentum space, we get the 'trivial' Ward identities which relate vertices via the manifest bosonic and broken fermionic gauge invariance. These identities provide a powerful check on solutions for $S$, and play a crucial rôle in the calculation to follow.

Applying the unbroken gauge symmetries (3.33):

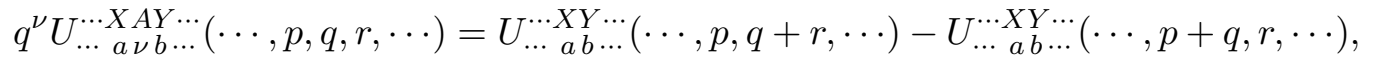

where $U$ stands for any element, i.e. a vertex from a covariantised kernel or from an action. $X_{a}$ and $Y_{b}$ are the fields $A, B, C$ or $D$, with $a$ and $b$ Lorentz indices or null as appropriate. Geometrically, the momentum of the gauge field is pushed forward along the direction of the Wilson line to the next 'obstruction' (with a plus) or pulled back against the direction of the Wilson line to the previous obstruction (and given a minus sign) [9. If $A$ is at the end of a line in a wine vertex, then either $X$ or $Y$ is $Z_{1}$ or $Z_{2}$ in the expansion (2.35) as appropriate, and the momentum is pushed forward (pulled back) onto this 110, 11]. Since $\sigma$ commutes with $\omega$, any $\sigma$ insertion is 'invisible' in this process and the momentum $q$ is pushed forward (pulled back) through the $\sigma$ position to the next 'real' obstruction. [This is also clear by temporarily (anti)commuting the $\sigma$ out of the way and then applying (3.35).]

Similarly, applying the broken supergauge symmetries (3.34), we get

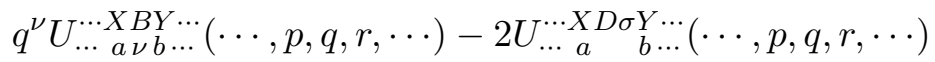

$$
\begin{aligned}
& =U_{\cdots X}^{\cdots X} \hat{Y} \cdots(\cdots, p, q+r, \cdots)-U_{\cdots}^{\cdots X} Y \cdots \cdots(\cdots, p+q, r, \cdots) .
\end{aligned}
$$

$X_{a}$ and $Y_{b}$ have the same interpretation as before. $\hat{X}$ and $\hat{Y}$ are the opposite statistics partners (thus $\hat{A}_{\mu}=B_{\mu}$, etc. ). This time, since $\tau$ anticommutes with $\sigma$, if the momentum $q$ is pushed back (pulled forward) through a $\sigma$ then the corresponding term on the right hand side of (3.36) changes sign. 


\section{Seed action two-point vertices}

As we have already emphasised, we do not restrict the seed action $\hat{S}$ to a particular choice. We will restrict the set of possible choices however, for technical reasons. For example in this paper we insist that $\hat{S}$ has only a single supertrace. We could have taken the form of the bare action from ref. [16] as one choice of seed action, but it is very helpful to add more general interactions than this, in order to avoid the appearance of certain flowing classical couplings ( $c f$. sec. 7.1). More importantly we now realise that it is to our advantage to keep $\hat{S}$, and the forms of covariantisation, general, since this guides us to an efficient procedure for calculation. Providing we are computing a well defined physical quantity, we are guaranteed that the result is independent of the detailed choices.

Consider first the $D D$ two-point vertex. ${ }^{14}$ By Goldstone's theorem [32], $D$ must be massless, thus by Lorentz invariance and dimensions its kinetic term takes the form

$$
\hat{S}^{D D}(p)=\Lambda^{2} p^{2} / \tilde{c}_{p}
$$

where $\tilde{c}_{p}=\tilde{c}\left(p^{2} / \Lambda^{2}\right)$ is a dimensionless smooth strictly positive function. (Recall from sec. 2.2 that $\Lambda$ is the only explicit scale that can appear, and smoothness is a requirement for all vertices.) Although it is not necessary [11], we set the kinetic term to be conventionally normalised, and so restrict our choices to $\tilde{c}(0)=1$.

Proceeding similarly, we have that in general there are two types of $A A$ vertex, however by (3.32), $\hat{S}_{\mu \nu}^{A A \sigma}=0$. From (3.35), the $A A$ vertex is totally transverse

$$
p^{\mu} \hat{S}_{\mu \nu}^{A A}(p)=0 .
$$

(Since $\operatorname{str} A=0$, single point $A$ vertices do not exist. ${ }^{15}$ ) By dimensions and Lorentz invariance, it therefore takes the form

$$
\hat{S}_{\mu \nu}^{A A}(p)=2 \square_{\mu \nu}(p) / c_{p},
$$

where $\square_{\mu \nu}(p) \equiv p^{2} \delta_{\mu \nu}-p_{\mu} p_{\nu}$ is the usual transverse kinetic term, which will appear often, and $c_{p}=c\left(p^{2} / \Lambda^{2}\right)$ is another dimensionless smooth strictly positive function. Recall from sec. 3 that we set the classical two-point vertex equal to this:

$$
S_{0 \mu \nu}^{A A}(p)=\hat{S}_{\mu \nu}^{A A}(p) .
$$

This implies from the renormalization condition (3.5), and (3.7), that $c(0)=1$. In order to maintain finiteness, we must limit the large momentum behaviour of $c$ and $\tilde{c}$, for power law large momentum behaviour as in (2.11).

This analysis shows that so far, apart from some very basic restrictions, the introduction of the cutoff functions merely parametrise the most general two-point vertices.

Now consider the $B B$ vertex. (Like $D D \sigma$, a $B B \sigma$ vertex cannot exist.) It has a transverse part, which by dimensions and Lorentz invariance has the same form as (4.3) but

\footnotetext{
${ }^{14}$ There is no $D D \sigma$ vertex since by $\{D, \sigma\}=0$ and cyclicity, $\operatorname{str} D D \sigma=-\operatorname{str} D \sigma D=-\operatorname{str} D D \sigma$.

${ }^{15}$ But also for many other reasons: Poincaré invariance, charge conjugation invariance, etc.
} 
with a possibly different cutoff function. However for $S U(N \mid N)$ invariance to be recovered at high energies, these cutoff functions must agree at high energies. For simplicity we just set them equal. (In the symmetric phase, $B$ and $A$ of course have the same kinetic term. In the broken phase they of course differ, for example from $\hat{S}^{\mathcal{A C} \mathcal{A C}} \mapsto \hat{S}^{\mathcal{A} \sigma \mathcal{A} \sigma}$. For the two kinetic terms to disagree also at high energies, these higher point interactions with $\mathcal{C}$ would have to have momentum dependence in the ultraviolet so violent as to destroy the higher derivative part of the regularisation of ref. [16.) The longitudinal part of the $B B$ vertex is already determined by two applications of broken fermionic gauge invariance (3.36):

$$
\begin{aligned}
p^{\mu} \hat{S}_{\mu \nu}^{B B}(p) & =-2 \hat{S}_{\nu}^{B D \sigma}(p) \\
p^{\mu} \hat{S}_{\mu}^{B D \sigma}(p) & =-2 \hat{S}^{D D}(p) .
\end{aligned}
$$

[In the first line we use cyclicity and then Lorentz invariance: $\hat{S}_{\nu}^{B D \sigma}(-p, p)=-\hat{S}_{\nu}^{B D \sigma}(p)$.] Thus

$$
\hat{S}_{\mu \nu}^{B B}(p)=2 c_{p}^{-1} \square_{\mu \nu}(p)+4 \Lambda^{2} \tilde{c}_{p}^{-1} \delta_{\mu \nu}
$$

and using (4.5),

$$
\hat{S}_{\mu}^{B D \sigma}(p)=-2 \Lambda^{2} p_{\mu} / \tilde{c}_{p}
$$

By using the fact that the vertex must be overall bosonic, and using charge conjugation [or the (broken super)gauge symmetries, or sometimes just (3.32)] one may readily show that all other mixed two-point vertices are disallowed.

Finally, we know from (3.32) that there is no $C C \sigma$ vertex. The difference between the $C C$ and $D D$ vertex, amounts to the addition of a new cutoff function that serves to give $C$ a mass, and thus must not vanish at $p=0$. In addition at high momentum it must be subleading compared to the $D D$ part in order that the symmetric phase be regained (as with $B B$ versus $A A$ ). For simplicity we simply choose it to be constant and thus,

$$
\hat{S}^{C C}(p)=\Lambda^{2} p^{2} / \tilde{c}_{p}+2 \lambda \Lambda^{4},
$$

where $\lambda>0$ is a constant parameter that is left undetermined.

This completes the parametrisation of the seed action two-point vertices. In point of fact they are the ones that would be obtained by setting the seed action to have the same form as the bare action of ref. [16], however we emphasise that the higher-point $\hat{S}$ vertices will not agree with the bare ones from [16]. These higher point vertices are constrained by the symmetries of the theory and most powerfully by (3.35, 3.36). By iterative use of these identities and the flow equations (3.9.3.10), we will be able to reduce the complete calculation of $\beta_{1}$ to a dependence only on the two-point vertices above. In this way, although we for simplicity set a number of restrictions (equality of cutoff functions for $B$ and $A, \tilde{c}(0)=1$, and that the mass term for $C$ is simply a constant), these come into play only at the end of the computation and could easily be lifted.

\section{The kernels}

From (3.2) and (2.34), the zero-point kernels in the broken phase take the form

$$
\dot{\Delta}^{A A}=\dot{\Delta}^{\mathcal{A A}}, \quad \dot{\Delta}^{B B}=\dot{\Delta}^{\mathcal{A A}}+\dot{\Delta}_{m}^{\mathcal{A A}}, \quad \dot{\Delta}^{C C}=\dot{\Delta}^{\mathcal{C C}} \quad \text { and } \quad \dot{\Delta}^{D D}=\dot{\Delta}^{\mathcal{C C}}+\dot{\Delta}_{m}^{\mathcal{C C}} .
$$


They will be represented graphically as in fig. 6, and are determined by the requirement
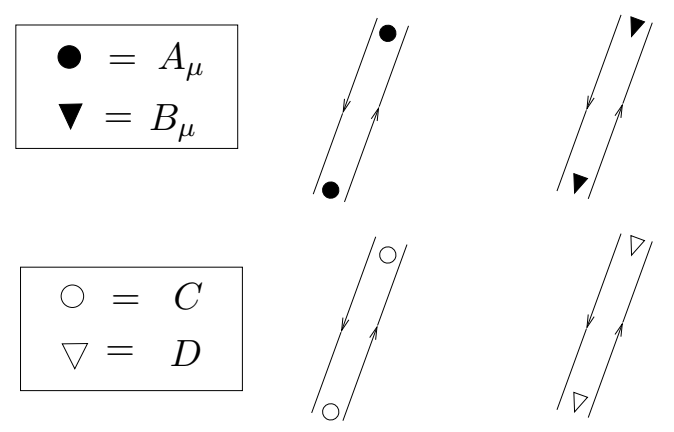

Figure 6: Graphical representation of 0-point wines

that the two-point vertices of classical effective action $S_{0}$ can be set equal to those of $\hat{S}$ ( $c f$. sec. 3). After setting $\hat{S}^{C C}=S_{0}^{C C}$, the flow equation for $S_{0}^{C C}$ takes the simple form given in fig. 7, giving

$$
\Lambda \partial_{\Lambda} S_{0}^{C C}(p)=S_{0}^{C C}(-p) \dot{\Delta}_{p}^{C C} S_{0}^{C C}(p)
$$

thus, since $\hat{S}^{C C}$ is an even function of $p$,

$$
\begin{aligned}
\dot{\Delta}_{p}^{C C} & =-\Lambda \partial_{\Lambda}\left(\hat{S}_{p}^{C C}\right)^{-1} \\
& =\frac{1}{\Lambda^{4}} \frac{1}{x}\left(\frac{2 x^{2} \tilde{c}}{x+2 \lambda \tilde{c}}\right)^{\prime},
\end{aligned}
$$

where here and later we use the notation, $x=p^{2} / \Lambda^{2}$, the cutoff terms being functions of this ratio, and prime denotes differentiation with respect to this.

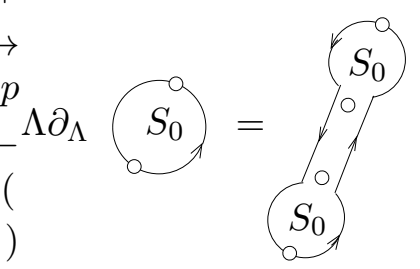

Figure 7: $C C$ tree-level equation. One $C$ has momentum $p$ and the other $-p$.

Similarly, using (4.4), the classical $A A$-point flow equation is

$$
\Lambda \partial_{\Lambda} \hat{S}_{\mu \nu}^{A A}(p)=\hat{S}_{\mu \alpha}^{A A}(p) \dot{\Delta}_{p}^{A A} \hat{S}_{\alpha \nu}^{A A}(p),
$$

If (4.3) were invertible, $\dot{\Delta}^{A A}$ would also take the form (5.3). Instead, substituting (4.3) we get:

$$
\dot{\Delta}^{A A}=c_{p}^{\prime} / \Lambda^{2}
$$

The $B B, D D$ and $B D \sigma$ classical flow equations are coupled, $c f$. e.g. fig. 8:

$$
\begin{aligned}
\Lambda \partial_{\Lambda} \hat{S}_{\mu \nu}^{B B} & =\hat{S}_{\mu \alpha}^{B B} \dot{\Delta}^{B B} \hat{S}_{\alpha \nu}^{B B}+\hat{S}_{\mu}^{B D \sigma} \dot{\Delta}^{D D} \hat{S}_{\nu}^{B D \sigma} \\
\Lambda \partial_{\Lambda} \hat{S}_{\mu}^{B D \sigma} & =\hat{S}_{\mu \alpha}^{B B} \dot{\Delta}^{B B} \hat{S}_{\alpha}^{B D \sigma}+\hat{S}_{\mu}^{B D \sigma} \dot{\Delta}^{D D} \hat{S}^{D D} \\
\Lambda \partial_{\Lambda} \hat{S}^{D D} & =\hat{S}_{\alpha}^{B D \sigma} \dot{\Delta}^{B B} \hat{S}_{\alpha}^{B D \sigma}+\hat{S}^{D D} \dot{\Delta}^{D D} \hat{S}^{D D}
\end{aligned}
$$


where $p$ is the momentum argument in all the above terms. These three equations are of course not independent: the last two are readily seen to follow from the first, on using the broken gauge transformations (4.5). By substituting (4.6) and (4.7) in the first (and isolating the transverse part or otherwise), it is straightforward to solve for the kernels:

$$
\begin{aligned}
& \dot{\Delta}^{B B}=-\Lambda \partial_{\Lambda}\left(2 p^{2} / c+4 \Lambda^{2} / \tilde{c}\right)^{-1}=\frac{1}{\Lambda^{2}}\left(\frac{x c \tilde{c}}{x \tilde{c}+2 c}\right)^{\prime} \\
& \dot{\Delta}^{D D}=-\Lambda \partial_{\Lambda}\left(\frac{\tilde{c}}{\Lambda^{2} p^{2}}\right)-\frac{4}{p^{2}} \dot{\Delta}^{B B}=\frac{1}{\Lambda^{4}} \frac{1}{x}\left(\frac{2 x^{2} \tilde{c}^{2}}{x \tilde{c}+2 c}\right)^{\prime} .
\end{aligned}
$$

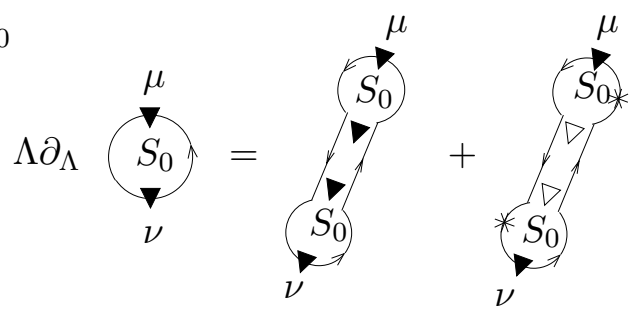

Figure 8: $B B$ tree-level equation.

From (5.1), the original kernels for (3.2, 3.3) and (2.34) are thus given by (5.4), (5.6), and

$$
\begin{aligned}
\dot{\Delta}_{m}^{\mathcal{A} \mathcal{A}} & =-\frac{1}{\Lambda^{2}}\left(\frac{2 c^{2}}{x \tilde{c}+2 c}\right)^{\prime} \\
\dot{\Delta}_{m}^{\mathcal{C C}} & =\frac{1}{\Lambda^{4}} \frac{1}{x}\left(\frac{4 x^{2} \tilde{c}\left(\lambda \tilde{c}^{2}-c\right)}{(x \tilde{c}+2 c)(x+2 \lambda \tilde{c})}\right)^{\prime} .
\end{aligned}
$$

Importantly, these mass-term type corrections to $\dot{\Delta}^{\mathcal{A A}}$ and $\dot{\Delta}^{\mathcal{C C}}$, which behave as expected from (4.6) and (4.8), decay much faster than (5.6) and (5.4), thus ensuring that at high momentum $p$, the exact RG (3.1) goes over to one appropriate for the symmetric phase of the $S U(N \mid N)$ theory. These corrections thus behave as required by the discussion below (2.11), in particular the $\mathcal{C}(+\sigma)$ decorations of (2.34), which destroy the supertrace mechanism, here can be taken to be regularised by the covariant higher derivatives alone.

\section{The integrated kernels}

By (3.11) and (5.3), we immediately see that the $C C$ integrated kernel is just the inverse kinetic term:

$$
\Delta^{C C} S_{0}^{C C}=1 \quad \Rightarrow \quad \Delta^{C C}=\frac{1}{\Lambda^{4}} \frac{\tilde{c}}{x+2 \lambda \tilde{c}}
$$

(choosing the integration constant here and later, so that the 'effective propagator' vanishes as $p \rightarrow \infty)$. We represent the integrated wine as in fig. 6, but with a line down its spine, and thus (6.1) is represented diagrammatically as in fig. 9 . 


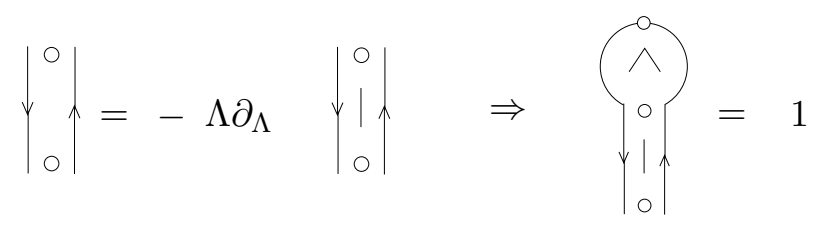

Figure 9: $C$ integrated wine. In this case there is no remainder.

Integrating (5.6), we have

$$
\Delta_{p}^{A A}=\frac{c_{p}}{2 p^{2}}
$$

Despite its similarity to a regularised Feynman propagator, we stress that no gauge fixing has taken place. Indeed this 'effective propagator' is the inverse of the classical $A A$ kinetic term only in the transverse space:

$$
\Delta_{p}^{A A} S_{0 \mu \nu}^{A A}(p)=\delta_{\mu \nu}-\frac{p_{\mu} p_{\nu}}{p^{2}}
$$

Since in practice $\Delta^{A A}$ will be connected to an $A$ point on some other vertex, the remainder term above will simply generate gauge transformations via (3.35). This observation proves crucial in the 'magic' of the calculation to follow.

The integrals via (3.11) of (5.8) and (5.9) are also immediate, and thus we find

$$
\begin{aligned}
\Delta^{B B} & =\frac{1}{2 \Lambda^{2}} \frac{c \tilde{c}}{x \tilde{c}+2 c} \\
\Delta^{D D} & =\frac{1}{\Lambda^{4}} \frac{\tilde{c}^{2}}{x \tilde{c}+2 c} .
\end{aligned}
$$

Note that despite the classical $D$ kinetic term being that of a massless (Goldstone) field, the $D$ effective propagator like that of $C$ and $B$ (but unlike $A$ ) has no massless pole. Of course this is nothing but the Higgs mechanism, arising here from the $B$ and $D$ two-point vertices being intimately entangled via (5.7). Similarly to the above reasoning, the pair of effective propagators (6.4) would form the inverse of the matrix of these fermionic twopoint vertices, if this matrix were invertible. It is not, for the same reason that these flows are necessarily entangled: $B$ and $D \sigma$ rotate into each other under the broken supergauge transformations (3.36).

\subsection{Five dimensions in the fermionic sector}

We thus need to consider $B$ and $D \sigma$ together. We write $B$ and $D \sigma$ as elements of a Euclidean 5 dimensional vector ${ }^{16}$

$$
F_{M}=\left(B_{\mu}, D \sigma\right)
$$

Introducing the '5-momentum'

$$
q_{M}=\left(q_{\mu},-2\right),
$$

\footnotetext{
${ }^{16}$ Note that this respects charge conjugation symmetry since $F_{M} \mapsto-F_{M}^{T}$.
} 
we see from (3.36) and (4.5), that the matrix $\hat{S}_{M N}^{F F}(p) \equiv \hat{S}_{M N}^{F F}(p,-p)$ is going to be transverse. Indeed, defining

$$
\hat{S}_{M N}^{F F}(p)=\left(\begin{array}{cc}
\hat{S}_{\mu \nu}^{B B}(p) & \hat{S}_{\mu}^{B D \sigma}(p) \\
-\hat{S}_{\nu}^{B D \sigma}(p) & -\hat{S}^{D D}(p)
\end{array}\right)
$$

where we have used $\hat{S}_{\nu}^{D \sigma B}(p)=-\hat{S}_{\nu}^{B D \sigma}(p)$ and $\hat{S}^{D \sigma D \sigma}=-\hat{S}^{D D}$, we have:

$$
p_{M} \hat{S}_{M N}^{F F}(p)=\hat{S}_{M N}^{F F}(p)(-p)_{N}=0 .
$$

Note that the argument of the 5-momentum is that of the 4-momentum inflow to the corresponding point, ${ }^{17}$ and by cyclicity the matrix is of course symmetric in this sense:

$$
\hat{S}_{M N}^{F F}(p)=\hat{S}_{N M}^{F F}(-p) .
$$

Wine attachments to $D$ must now attach to $D \sigma$. The result is an extra factor of $(-)^{1+f_{\sigma}}$, where $f_{\sigma}=0(1)$ if either side of the wine is bosonic (fermionic), as is clear from fig. 10 .

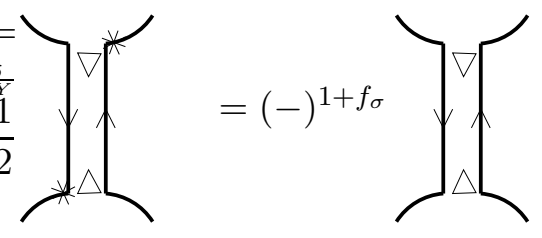

Figure 10: Wine attachment to $D \sigma$.

Thus the fermionic effective propagators (6.4) collect into:

$$
\Delta_{M N}^{F F}(p)=\left(\begin{array}{cc}
\Delta_{p}^{B B} \delta_{\mu \nu} & 0 \\
0 & -\Delta_{p}^{D D}
\end{array}\right),
$$

and $\dot{\Delta}_{M N}^{F F}$ is simply the differential of this, according to 3.11 ). Apart from these extra factors (and the need to add 5-indices $M$ and $N$ under the flavour labels) the tensorial expansions (2.35) and (2.38) map unchanged to five dimensional notation. In this way, all equations relating to the fermionic sector can be written in this language, where they take a compact form very similar in appearance to the corresponding expressions for $A$ and $C$. For example from (5.7), $\Lambda \partial_{\Lambda} \hat{S}_{M N}^{F F}=\hat{S}_{M A}^{F F} \dot{\Delta}_{A B}^{F F} \hat{S}_{B N}^{F F}$, cf. (5.2) and (5.5), and from (3.36), broken fermionic gauge transformations now map exclusively to lower point vertices as [compare (3.35)]:

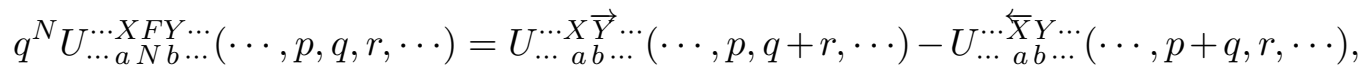

where on the elementary fields $X=A, B, C, D, \overleftarrow{X}=\vec{X}=\hat{X}$ is just the opposite statistics partner. Similarly, $\vec{F}_{M}=\left(A_{\mu}, C \sigma\right)$, but $\vec{F}_{M}=\left(A_{\mu},-C \sigma\right)$, the extra sign being picked up by $\tau$ anticommuting through $\sigma$. As for (3.36), there are compensating signs on the right hand side for any other $\sigma$ that the momentum is pushed through.

\footnotetext{
${ }^{17}$ N.B. $(-p){ }_{M} \neq-p_{M}$.
} 
Finally, multiplying $(6.7)$ and $(6.10)$ one readily finds the pair [related by transposition as in $(6.9)$ ]:

$$
\begin{aligned}
& \hat{S}_{R S}^{F F}(p) \Delta_{S T}^{F F}(p)=\delta_{R T}-p_{R}^{\prime} p_{T} \\
& \Delta_{R S}^{F F}(p) \hat{S}_{S T}^{F F}(p)=\delta_{R T}-(-p)_{R}(-p)_{T}^{\prime},
\end{aligned}
$$

where introducing the useful shorthands

$$
f=\frac{\tilde{c}}{x \tilde{c}+2 c} \quad \text { and } \quad g=\frac{c}{x \tilde{c}+2 c},
$$

we define a dual 5-momentum $p_{M}^{\prime}$ as follows

$$
p_{M}^{\prime}=\left(f p_{\mu} / \Lambda^{2},-g\right)
$$

Note that since $2 g+x f=1$, we have $p_{M}^{\prime} p_{M}=1$, and thus (6.12) and (6.13) are projectors onto the appropriate transverse space, just as in (6.3). Furthermore, we see that since in practice $\Delta^{F F}$ will be connected to an $F$ point on some other vertex, the remainder from unity in (6.12,6.13) always generates supergauge transformations via (6.11).

\section{Guaranteeing universality of $\beta_{1}$ (and $\beta_{2}$ )}

We now review in this context, the standard argument for why we should expect to get the same value for $\beta_{1}$, and indeed $\beta_{2}$, in the $\beta$ function (3.8) as in other methods, despite the fact that our renormalization scheme for $g(\Lambda)$ differs from that of the corresponding coupling $\tilde{g}(\mu \mapsto \Lambda)$ defined by these other methods. (We note that the Gribov problem 13] which in truth invalidates these methods since they proceed by gauge fixing, is not expected to alter purely perturbative results.)

In principle we can extract from (3.7), by computing quantum corrections, the value of the other coupling as a function of ours, and thus match the two couplings perturbatively:

$$
1 / \tilde{g}^{2}=1 / g^{2}+\gamma+O\left(g^{2}\right)
$$

where the classical agreement is guaranteed by the standard normalisations of the fields and kinetic term in (3.5), after scaling $g$ back to its usual position, and $\gamma$ is a one-loop matching coefficient. Differentiating with respect to $\Lambda$ and using (3.8), the corresponding $\beta$ function for $\tilde{g}$, and (7.1), we have

$$
\tilde{\beta}_{1}+\tilde{\beta}_{2} g^{2}=\beta_{1}+\beta_{2} g^{2}+\Lambda \partial_{\Lambda} \gamma+O\left(g^{4}\right) .
$$

Since $\gamma$ is dimensionless, it cannot depend upon $\Lambda$, there being no other scale to form the necessary dimensionless combination. Thus $\Lambda \partial_{\Lambda} \gamma=0$ in (7.2), and we immediately recover the standard facts that $\tilde{\beta}_{1}=\beta_{1}$ and $\tilde{\beta}_{2}=\beta_{2}$.

Clearly this argument fails if some other scale has been introduced, for example the standard arbitrary finite physical scale $\mu$, or if other running couplings get introduced. (After solving for their flows, i.e. solving their corresponding $\beta$ functions, this becomes 
equivalent to the first failure since by dimensional transmutation a new finite physical scale has been introduced.) Importantly, $\Lambda \partial_{\Lambda} \gamma$ can then have an $O\left(g^{2}\right)$ one-loop contribution or in extreme cases even a tree-level $O\left(g^{0}\right)$ contribution. From (7.2) one sees that a one-loop contribution to the running of $\gamma$ destroys $\beta_{2}$ agreement, whilst a tree-level running would even modify $\beta_{1}$.

As we will see shortly, a generic $\hat{S}$, including the simple form used for the bare action in ref. [16], can lead to such tree-level corrections. Fortunately, there is also an infinite class of seed actions that cannot. As with the earlier constraints discussed, since we never specify $\hat{S}$, it is not the solution that matters, only knowing that one exists.

To get agreement with the standard $\beta$ function at the two-loop level, one needs to confirm that there are no further couplings hidden, that run at one loop, and to take into account contributions from $g_{2}(\Lambda)$. This can be done [1]].

Even with a non-vanishing $\Lambda \partial_{\Lambda} \gamma$, one could still recover the usual $\beta$ function coefficients, by defining a standard low energy -or infrared-coupling $\tilde{g}(\mu)$ at some scale $\mu<\Lambda$, this coupling being distinguished from the 'ultraviolet' coupling $g(\Lambda)$ in the effective action $S_{\Lambda}$ [33, 34]. We want to avoid this because the introduction of $\mu$ would destroy, or at least obscure, the power and elegance of self-similarity [27] ( $c f$. sec. 2.2).

\subsection{Ensuring no running couplings at tree level}

The incorporation of Pauli-Villars type fields directly into an exact RG causes some novel classical divergences in the $\Lambda$ integrals defining the classical vertices, just as they did in ref. [11, 9]. They can be cancelled by appropriate choices of integration constant. However, generically this results in introducing another finite scale $\mu$, even at the classical level, again just as it did in ref. [11]. The resulting loss of self-similarity leads to non-universal contributions creeping in at a particular point in the calculation of $\beta_{1}$ that follows. Indeed, we will see there that it is precisely the classical dependence on $\mu$ that causes the problem.

To show how this arises, and how we can avoid it, consider the classical $C C A$ vertex. This is one of a number of affected vertices that form part of the $\beta_{1}$ calculation. The flow follows from eq. (3.9), and is given by the first four diagrams on the right hand side of fig. 13 after replacing the star by an open circle. The resulting equation, up to changes of notation, is precisely equivalent to the $C C A$ vertex in ref. [11]:

$$
\begin{aligned}
S_{0 \mu}^{A C C}(p, q, r)=-\int_{\Lambda} \frac{\Lambda_{0}}{d \Lambda_{1}} & \hat{S}_{\mu}^{A C C}(p, q, r)\left[\dot{\Delta}_{q}^{C C} \hat{S}_{q}^{C C}+\dot{\Delta}_{r}^{C C} \hat{S}_{r}^{C C}\right] \\
& \left.\quad+\hat{S}_{\alpha}^{A C C}(p, q, r) \dot{\Delta}_{p}^{A A} \hat{S}_{\alpha \mu}^{A A}(p)+\hat{S}_{r}^{C C} \dot{\Delta}_{\mu}^{A, C C}(p ; r, q) \hat{S}_{q}^{C C}\right\} \\
& +\Lambda_{0}^{2}(q-r)_{\mu}+\tilde{c}_{0}^{\prime}\left(q^{2}+r^{2}\right)(r-q)_{\mu}+\gamma^{A C C} q_{\alpha} \square_{\alpha \mu}(p),
\end{aligned}
$$

where all the terms inside the curly brackets are to be understood as being functions of $\Lambda_{1}$ (not $\Lambda$ ), and $\tilde{c}_{0}^{\prime} \equiv \tilde{c}^{\prime}(0)$. Here we have recognized that we can integrate the flow equation immediately with respect to $\Lambda$, but to make explicit the divergences we have replaced the upper limit by $\Lambda_{0}$. The integration constant ensures however that the complete expression is finite, so the continuum limit $\Lambda_{0} \rightarrow \infty$ can actually be safely taken. 
The first two terms in the integration constant, are forced by gauge invariance [1]. Indeed, setting $\Lambda=\Lambda_{0}$ in (7.3), we see that the integration constant is nothing but the $A C C$ vertex of the classical bare action $\left.S_{0}\right|_{\Lambda=\Lambda_{0}}$. Its longitudinal part follows from (3.35) and (4.8) which implies,

$$
p^{\mu} S_{0 \mu}^{A C C}(p, q, r)=\hat{S}_{r}^{C C}-\hat{S}_{q}^{C C} .
$$

This equation is readily solved at $\Lambda=\Lambda_{0}$, by expanding both sides as a power series in $\Lambda_{0}$, and noting that all negative powers can be discarded. ${ }^{18}$ Equivalently and more simply, the longitudinal terms follow from any covariantisation of (4.8), e.g.

$$
\frac{1}{2} \operatorname{str} \int d^{4} x C\left\{2 \lambda \Lambda_{0}^{4}-\Lambda_{0}^{2} D_{\mu}^{2}+\tilde{c}_{0}^{\prime}\left[D_{\mu}^{2}\right]^{2}+O\left(1 / \Lambda_{0}^{2}\right)\right\} C+\cdots
$$

(Recall that $D_{\mu}$ is $\partial_{\mu}-i A_{\mu}$ and acts by commutation. The ellipsis refers to terms not containing $C C A$ vertices.)

The final term in the integration constant in (7.3), $q_{\alpha} \square_{\alpha \mu}(p)$, is the unique transverse combination that is allowed by dimensions (i.e. is not accompanied by a negative power of $\Lambda_{0}$, and importantly satisfies all the other symmetries specifically charge conjugation and no- $\mathcal{A}^{0}$ symmetry), and as such has an undetermined (dimensionless) coefficient: $\gamma^{A C C}$. The fact that it is undetermined does not matter: the whole calculation is independent of such details. However, by the same token $\zeta^{A C C}$, the coefficient of this momentum term in the small momentum (a.k.a. derivative) expansion of the integrand in (7.3), is also dimensionless, thus independent of $\Lambda_{1}$, and thus yields $-\zeta^{A C C} \ln \left(\Lambda_{0} / \Lambda\right)$ on integration. In order to ensure the finiteness of (7.3), we are forced to introduce a new finite physical scale by including $\zeta^{A C C} \ln \left(\Lambda_{0} / \mu\right)$ in the integration constant $\gamma^{A C C}$.

An alternative to introducing $\mu$ directly, is to allow $\gamma^{A C C}$ to become a logarithmically running coupling $\gamma^{A C C}(\Lambda)$ at the classical level, correcting the flow to take account of its $\beta$ function. However as we explained in the previous section, this is in the end equivalent and still results in the loss of universality for $\beta_{1}$.

In general we see that we may be forced to introduce $\mu$ at the classical level wherever a purely local vertex with dimensionless coupling can be constructed, which is transverse, in the sense that it is unrelated to lower point vertices via either bosonic gauge invariance (3.35), or the broken fermionic gauge invariance (3.36).

We note that the problem is associated with the Pauli-Villars sector because these terms necessarily have a divergent classical action as $\Lambda \rightarrow \infty$, at least in so far as they have divergent masses. There is a problem with this only for the generated logarithmic divergences along the marginal directions however, whose cancellation necessarily requires introducing a new finite physical scale. There are however infinitely many of these directions because we can have any number of $C$ points, since $C$ is dimensionless.

The solution is to tune the corresponding terms in $\hat{S}$. Indeed by noting from (5.3), that $\dot{\Delta}^{C C} \Delta^{C C}=\Lambda \partial_{\Lambda} \ln \hat{S}^{C C}$ and thus equals 4 at zero momentum, we get from (7.3) that the shift

$$
\hat{S}_{\mu}^{A C C}(p, q, r) \mapsto \hat{S}_{\mu}^{A C C}-\frac{1}{8} \zeta^{A C C} q_{\alpha} \square_{\alpha \mu}(p)
$$

\footnotetext{
${ }^{18}$ The coefficients are purely local, i.e. polynomials in momenta, with determined dimension, $c f$. sec. 2.2.
} 
precisely cancels the coefficient of $q_{\alpha} \square_{\alpha \mu}(p)$ in the integrand, thus removing the logarithmic divergence from the $\Lambda_{1}$ integral.

Since the structure of the classical flow equations (3.9) is such, that the flow of every vertex $S_{0 a_{1} \cdots a_{n}}^{X_{1} \cdots X_{n}}$ has the corresponding $\hat{S}_{a_{1} \cdots a_{n}}^{X_{1} \cdots X_{n}}$ as its highest-point $\hat{S}$ contribution, contracted via kernels into all the appropriate two point vertices (viz. $\dot{\Delta}^{X_{i} X_{i}} \hat{S}^{X_{i} X_{i}}$ ) [10, 11], and since these $\dot{\Delta}^{X_{i} X_{i}} \hat{S}^{X_{i} X_{i}}$ terms are non-vanishing at zero momentum precisely when $X_{i}$ is a massive Pauli-Villars field, it follows that we can always remove the divergence associated with these marginal directions by tuning $\hat{S}_{a_{1} \cdots a_{n}}^{X_{1} \cdots X_{n}}$ in the same direction.

In this way, we completely avoid introducing $\mu$ (equivalently marginal running couplings) at the classical level.

\section{The calculation}

The whole of the paper up to this point has been concerned with setting up and justifying the formalism we will now use. We can now finally turn to computation itself.

The renormalization condition (3.5) constrains the two- $A$ point vertices, and by (4.3) and (4.4), this constraint is already saturated at tree level:

$$
S_{\mu \nu}^{A A}(p)+S_{\mu \nu}^{A A \sigma}(p)=\frac{2}{g^{2}} \square_{\mu \nu}(p)+O\left(p^{3}\right)=\frac{1}{g^{2}} S_{0 \mu \nu}^{A A}(p)+O\left(p^{3}\right) .
$$

It follows that all higher loop contibutions, $S_{n \mu \nu}^{A A}(p)+S_{n \mu \nu}^{A A \sigma}(p)$, must vanish at $O\left(p^{2}\right)$. From the discussion below (3.32), the one-loop contribution is purely of form $S_{1 \mu \nu}^{A A \sigma}$. (Thus it is already clear that $g_{2}$ has the opposite sign $\beta_{1}$, consistent with the wrong sign action in (3.5) 11]. This will be fully developed in ref. [1].) Specialising the one-loop flow equation (3.10) to two $A$ s and $O\left(p^{2}\right)$, and using (4.4), we see that it collapses to the purely algebraic relation [10, 11]:

$$
-2 \beta_{1} S_{0 \mu \nu}^{A A}(p)+O\left(p^{3}\right)=a_{1}\left[\Sigma_{0}\right]_{\mu \nu}^{A A \sigma}(p) .
$$

( $\Sigma_{0}=S_{0}-2 \hat{S}$.) Diagrammatically, this takes the form of fig. 11, after including the factor 2 from the two different supertraces that $A A$ can go into ( $c f$. end of sec. 3.2), and a factor 2 from adding the contribution $p_{\mu} \leftrightarrow-p_{\nu}$ (which is equal by Lorentz invariance. Whenever such terms arise we will typically combine them.)

$$
-4 \beta_{1} \square_{\mu \nu}(p)+O\left(p^{3}\right)=2 \sum_{f=A, C, F}[\underbrace{\Sigma_{0}}_{\nu}+\underbrace{\mu}_{\nu}+\underbrace{\Sigma_{0}}_{\nu}]
$$

Figure 11: Graphical representation of the equation for $\beta_{1}$

Performing the $\sigma$ algebra as in fig. 5, we thus find

$$
-4 \beta_{1} \square_{\mu \nu}(p)+O\left(p^{3}\right)=2 N \int \frac{d^{4} k}{(2 \pi)^{4}}\{
$$




$$
\begin{aligned}
& \dot{\Delta}_{k}^{C C} \Sigma_{0 \mu \nu}^{A A C C}(p,-p, k,-k)+\dot{\Delta}_{\mu}^{A, C C}(p ;-k-p, k) \Sigma_{0 \nu}^{A C C}(-p, p+k,-k) \\
& \quad+\dot{\Delta}_{\mu \nu}^{A A, C C}(p,-p ;-k, k) \Sigma_{0}^{C C}(k) \\
& +\dot{\Delta}_{k}^{A A} \Sigma_{0 \alpha \alpha \mu \nu}^{A A A A}(k,-k, p,-p)+\dot{\Delta}_{\mu}^{A, A A}(p ;-k-p, k) \Sigma_{0 \nu \alpha \alpha}^{A A A}(-p, p+k,-k) \\
& \quad+\dot{\Delta}_{\mu \nu}^{A A, A A}(p,-p ;-k, k) \Sigma_{0 \alpha \alpha}^{A A}(k) \\
& -\dot{\Delta}_{S R}^{F F}(k) \Sigma_{0 \mu \nu R S}^{A A F F}(p,-p, k,-k)-\dot{\Delta}_{\mu, S R}^{A, F F}(p ;-k-p, k) \Sigma_{0 \nu R S}^{A F F}(-p, p+k,-k) \\
& \left.\quad-\dot{\Delta}_{\mu \nu, S R}^{A A, F F}(p,-p ;-k, k) \Sigma_{0}^{F F}(k)\right\} .
\end{aligned}
$$

Although extra $\sigma$ s appear via fig. 5, the part that contributes from the $\sigma$ trapped in the empty supertrace (thus giving $\operatorname{str} \sigma=2 N$ ) ultimately comes from the breaking of $S U(N \mid N)$, i.e. from shifting $\mathcal{C}$, since otherwise the fermionic and bosonic attachments would just give equal and opposite $\sigma$ contributions in fig. 5, combining to give a full supermatrix differential. Indeed at high momentum $k$, exact $S U(N \mid N)$ invariance is recovered, resulting in regularisation of (8.3), since the $F$ sector then cancels the $A, C$ sector.

Recall that we exclude diagrams where the wine bites its own tail, as in 2.37). In fact such terms vanish for $\beta_{1}$ in any case, since the attachments are via a full $\mathcal{A}$ or $\mathcal{C}$ in (2.34) with no possibility of trapping an extra $\sigma$, thus yielding str $\mathbb{1}=0$.

The $\beta_{1}$ computation splits into one-loop contributions from the three sectors $C, A$, and $F$, each of which appears in (8.3) in almost identical form. Thus apart from the sign, we get the $A$ sector terms from the $F$ sector simply by replacing $F$ by $A$, and $R, S$ by $\alpha, \beta$, recognizing that the wines just have $\delta_{\alpha \beta}$ as a factor. Similarly, we get the $C$ contribution from the $F$ contribution by $F \mapsto C$, dropping $S$ and $R$ altogether.

\subsection{Diagrammatic analysis}

The similarity goes deeper. The $F$ type classical four-point vertex in (8.3) is determined by the flow equation given diagrammatically in fig. 12, whilst the $F$ type classical three-point vertex (appearing in (8.3) and fig. 12) is expressed through the flow equation of fig. 13 . The corresponding $A(C)$ sector diagrams are given simply by replacing the star with a filled (empty) circle!

This works because all cyclically allowed configurations of the external fields appear in fig. 12 and fig. 13, after which the flavour of the point the wine attaches to, is uniquely determined by the requirement that each component vertex has an even number of stars, so as to be overall bosonic. For the $A$ sector the fact that there is just one wine attachment for each external configuration is enough to ensure the mapping works. For the $C$ sector, the symmetry (3.32) ensures that each vertex also has an even number of open circles, which thus go uniquely where the stars had gone before. There are a couple of provisos however. Firstly, all the wines which attach via an $F$ at one end and an $A$ at the other, map under $F \mapsto C$, to wines that do not exist in (3.2). This does not matter: we can simply assign them a zero value, and carry these vanishing terms through the computation. (There is no fundamental reason for their non-appearance: recall that we exchanged such terms for simpler terms using (3.15). Also note that actually the $F$ attachment here is exclusively a $B$. When we take this into account, it will be clear that the term only has a mapping to the $A$ sector.) Secondly (3.32) in fact also allows an odd number of $C$ 's per classical vertex 


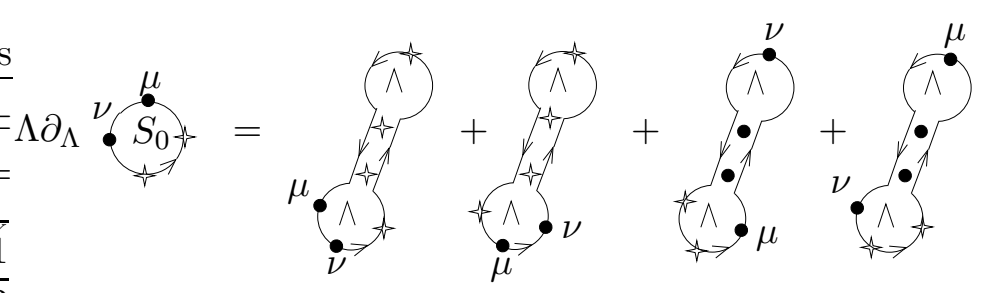
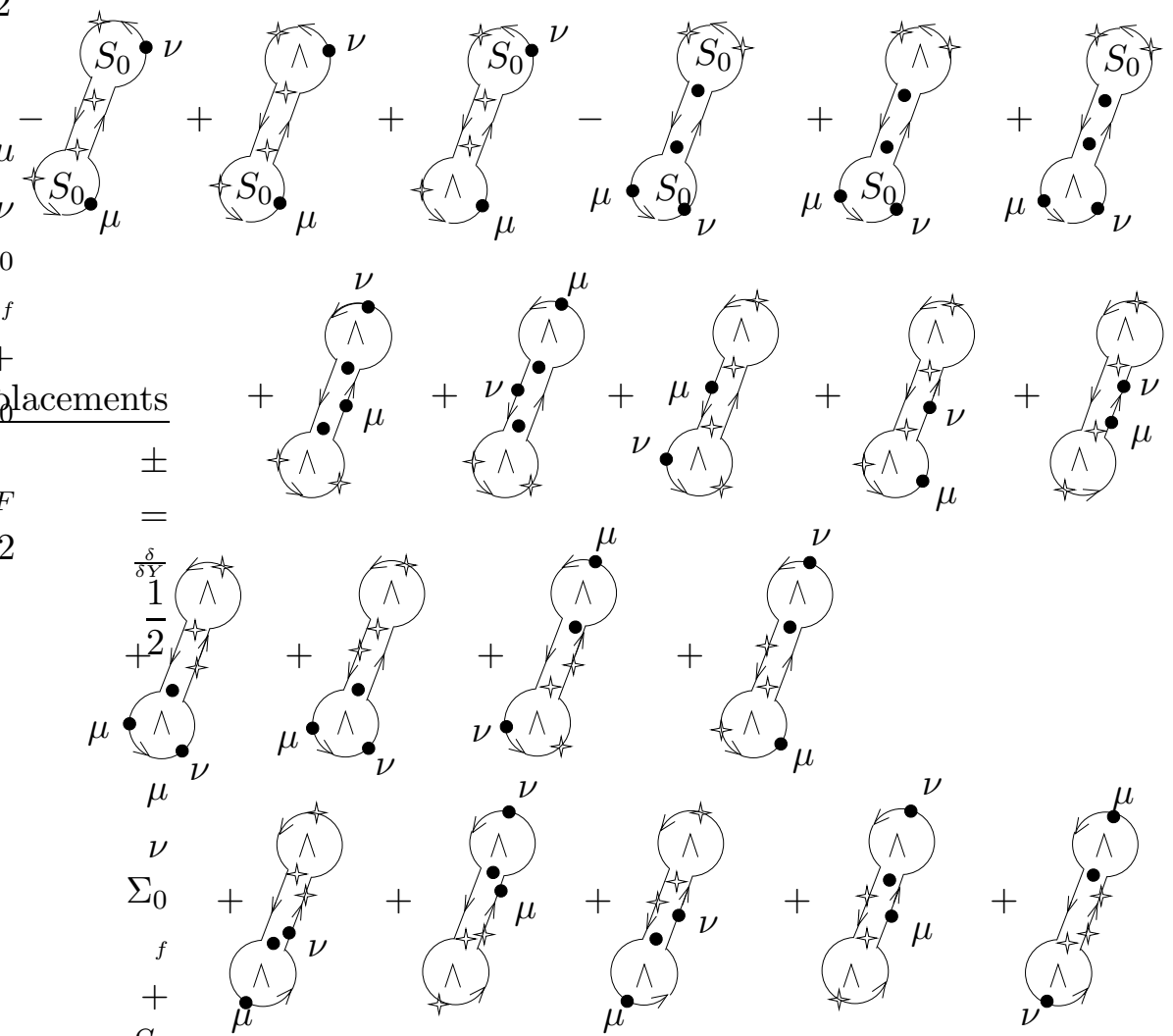

Figure 12: Flow of the tree-level $A A F F$ vertex. $F$ is represented by the star.

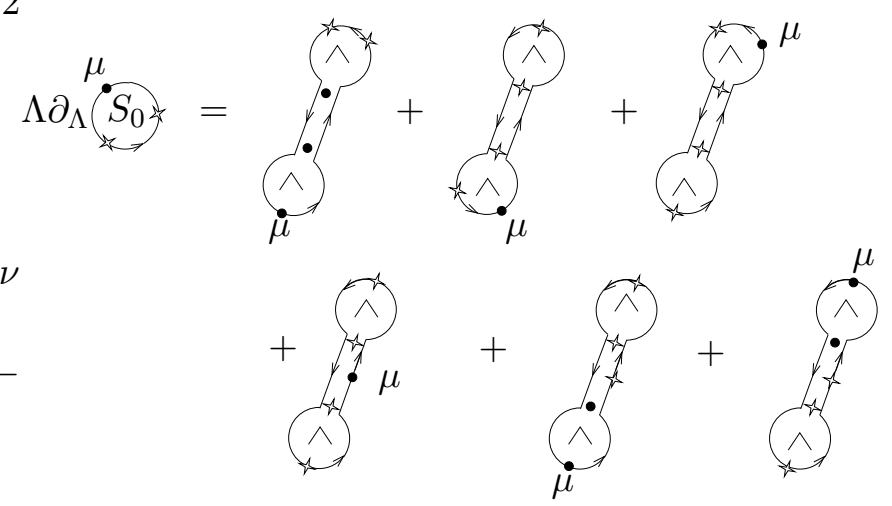

Figure 13: Flow equation for the tree-level $F F A$ vertex 
in the $C$ sector, providing that the vertex also has a $\sigma$. But in the case of the wines, these terms vanish by (2.34) since the $\sigma$ commutes with the bosonic $A$ or $C$ derivative, whilst the only action vertex that could contribute is $A A C \sigma$ ( $A C \sigma$ being already excluded by symmetries $c f$. sec. 1 , and all other possibilities being too high order). However, by charge conjugation invariance

$$
S_{\mu \nu}^{A A C \sigma}(p, q, r)=S_{\nu \mu}^{A A C \sigma}(q, p, r),
$$

while so long as we insist on a single supertrace, by no- $\mathcal{A}^{0}$ symmetry:

$$
S_{\mu \nu}^{A A C \sigma}(p, q, r)+S_{\nu \mu}^{A A C \sigma}(q, p, r)=0,
$$

thus the vertex actually vanishes. (Allowing multiple supertrace terms, it can be shown that the part contributing to $\beta_{1}$ vanishes after ensuring no running classical couplings as in sec. 7.1.)

We can map the effective propagator relations $(6.12,6.13$ ) in an obvious way to the corresponding relation for $A$, viz. (6.3), and $C$, viz. (6.1), leaving the gauge remainder terms in the case of $F$ and $A$ till last (where the $A$ sector expression follows from the map $k_{N} \mapsto k_{\nu}, k_{M}^{\prime} \mapsto k_{\mu} / k^{2}$, and of course in the $C$ sector these terms map to zero). This all means that we have an added bonus: except for these final stages, we need only present the $F$ sector computation and then just map the result to the other two sectors.

Substituting $\Sigma_{0}=S_{0}-2 \hat{S}$ in fig. 11, we start with the highest point vertex and convert the $S_{0}$ part to a total $\Lambda$ derivative and remainder, using (3.11):

$$
\begin{aligned}
\dot{\Delta}_{S R}^{F F}(k) S_{0 \mu \nu R S}^{A A F F}(p,-p, k,-k)= & -\Lambda \partial_{\Lambda}\left[\Delta_{S R}^{F F}(k) S_{0 \mu \nu R S}^{A A F F}(p,-p, k,-k)\right] \\
& +\Delta_{S R}^{F F}(k) \Lambda \partial_{\Lambda} S_{0 \mu \nu R S}^{A A F F}(p,-p, k,-k),
\end{aligned}
$$

as shown diagrammatically in fig. 14 .

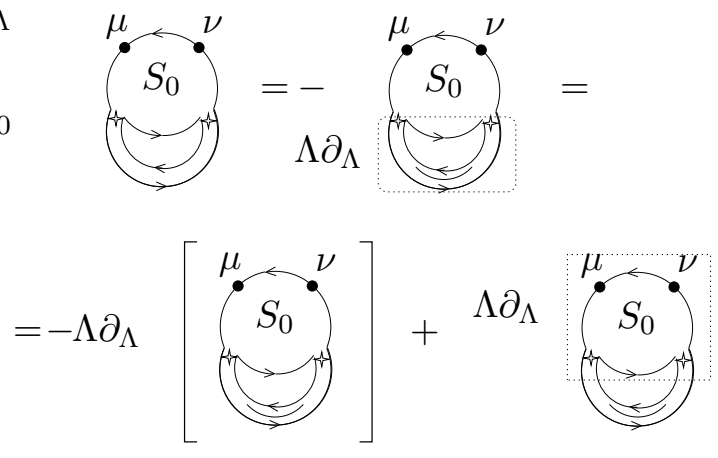

Figure 14: The first step in the calculation of $\beta_{1}$.

In the first term, we will put the $\Lambda \partial_{\Lambda}$ outside the $k$ integral. If the integral were convergent, the part we want, namely the $O\left(p^{2}\right)$ part, would then vanish since it is a dimensionless function of the only scale $\Lambda$. After adding in the $A$ and $C$ sectors, the integral will only have an infrared divergence, which $\Lambda \partial_{\Lambda}$ converts to a universal contribution.

In the second term we can now exchange the four-point vertex for lower point vertices via fig. 12: the generated four-point $\hat{S}$ vertices must cancel the $-2 \hat{S}_{\mu \nu R S}^{A A F F}$ in (8.3) in order 
for the result to be universal. This must be so because $\hat{S}^{A A F F}$ can contain arbitrary transverse terms, which thus have no relation to lower point vertices. Such a cancellation is precisely what we find from the first two diagrams in fig. 12, on using (6.13), as we can see from fig. 15. The remainder term from (6.13) generates a gauge transformation via

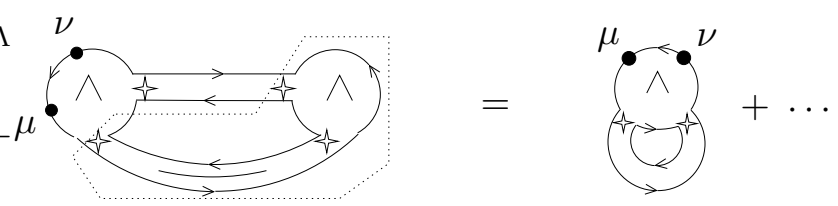

Figure 15: One of the pair cancelling $-2 \hat{S}^{A A F F}$ in fig. 11. The ellipsis are the gauge remainder terms.

(6.11) and thus also maps to lower point vertices. We will return to these gauge remainder terms later.

We are still left with two terms containing a four-point vertex, that of fig. 16, as generated by the next two diagrams in fig. 12. However now note, as we will frequently, that the two-point $A$ vertex is already $O\left(p^{2}\right)$, by gauge invariance $c f$. (4.3). Therefore the only part that can contribute to (8.3) is where $p$ is set to zero in the rest of the expression. After using Lorentz invariance to exchange $p_{\mu}$ and $-p_{\nu}$ in the second diagram, this results in a contribution

$$
-\Delta_{S R}^{F F}(k)\left[\hat{S}_{\mu \alpha R S}^{A A F F}(0,0, k,-k)+\hat{S}_{\alpha \mu R S}^{A A F F}(0,0, k,-k)\right] \dot{\Delta}_{0}^{A A} \hat{S}_{\alpha \nu}^{A A}(p)
$$

to the integrand in (8.3). As soon as we have an $A$-point with zero momentum, we can simplify via gauge invariance. Using (3.35) twice over, we have:

$$
\hat{S}_{\mu \nu R S}^{A A F F}(-\epsilon, \epsilon, k,-k) \epsilon^{\mu} \epsilon^{\nu}=\hat{S}_{R S}^{F F}(k+\epsilon)-\hat{S}_{\mu R S}^{A F F}(0, k,-k) \epsilon^{\mu}-\hat{S}_{R S}^{F F}(k) .
$$

Thus, Taylor expanding to $O\left(\epsilon^{2}\right)$, we determine the symmetric part:

$$
\hat{S}_{\mu \nu R S}^{A A F F}(0,0, k,-k)+\hat{S}_{\nu \mu R S}^{A A F F}(0,0, k,-k)=\partial_{\mu}^{k} \partial_{\nu}^{k} \hat{S}_{R S}^{F F}(k) .
$$

Substituting this in (8.7) we reduce fig. 16 to an expression depending only on seed action two-point vertices and their associated zero-point kernels (integrated or otherwise). We will refer to such terms as 'potentially universal', since the seed action two-point vertices and the kernels derived from them are the only things that we have explicitly prescribed. For the result to be universal, it must be that we can reduce everything to such potentially universal terms or to total $\Lambda$ derivatives as in (8.6). In turn, potentially universal terms must, and do, collect into total $k$ derivatives, whose boundary terms on integration, are universal as a result of restrictions on the large momentum behaviour, e.g. (2.11), and the renormalization condition (3.5). (Actually, since $\dot{\Delta}_{0}^{A A} \propto c_{0}^{\prime}$, by (5.6), and $1 / c_{0}^{\prime}$ is never produced, terms such as (8.7) are universal only because they combine to give boundary terms that vanish.)

Proceeding with the remaining terms in fig. 12, we generate many further reductions similar to the ones above. 


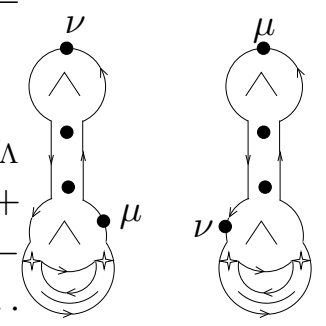

Figure 16: The two remaining terms containing a four-point vertex.

There are eight terms that immediately have vanishing $O\left(p^{2}\right)$ component. Two examples are shown in fig. 17. By Lorentz invariance, the first diagram has only odd powers of $p$, its dependence coming from $\hat{S}_{\mu \nu \alpha}^{A A A}(p,-p, 0)$, whilst the second diagram is also too high order since $\dot{\Delta}_{\mu}^{A, A A}(0 ; 0,0)=0$. Finally, the last term from fig. 12 is obviously $O\left(p^{4}\right)$.

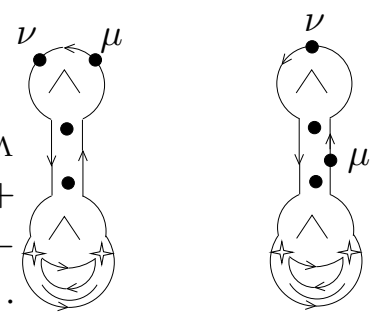

Figure 17: These diagrams do not contribute at $O\left(p^{2}\right)$

After using (6.12,6.13), a mirror pair (i.e. related by mirror reflection, equivalent to charge conjugation) cancel the seed action part of $\Sigma_{0}^{A F F}$, as shown in fig. 18, generating further gauge remainder terms. One term cancels the final $\Sigma_{0}^{F F}=-\hat{S}^{F F}$ term in (8.3), as

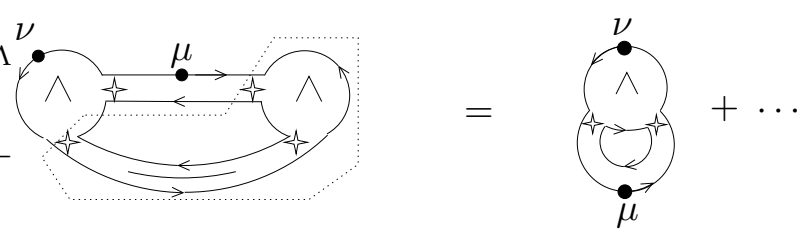

Figure 18: One of the pair cancelling $-2 \hat{S}^{A F F}$ in fig. 11 .

shown in fig. 19. Actually, here there is no point in carrying forward the gauge remainder, since it is clear by (6.13) and (6.8), and by (6.3) and (4.2), that it vanishes for both $F$ and $A$ sectors.

From the second line of fig. 12, there are three terms generated where the integrated kernel attaches to two different three-point vertices. These are either both $S_{0}$ vertices, or one $S_{0}$ vertex and one $\hat{S}$ vertex. They simplify after introducing a second integrated kernel into the $S_{0}-S_{0}$ term, as in fig. 20 (the third step following after using $p_{\mu} \leftrightarrow-p_{\nu}$ and relabelling the loop momentum). 


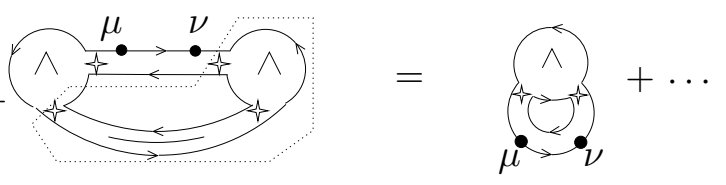

Figure 19: Cancellation of $-\hat{S}^{F F}$ in fig. 11.

The total $\Lambda$ derivative will be considered along with that of fig. 14. The other two terms are evaluated by substituting fig. 13 and its $p_{\mu} \mapsto-p_{\nu}$ partner.

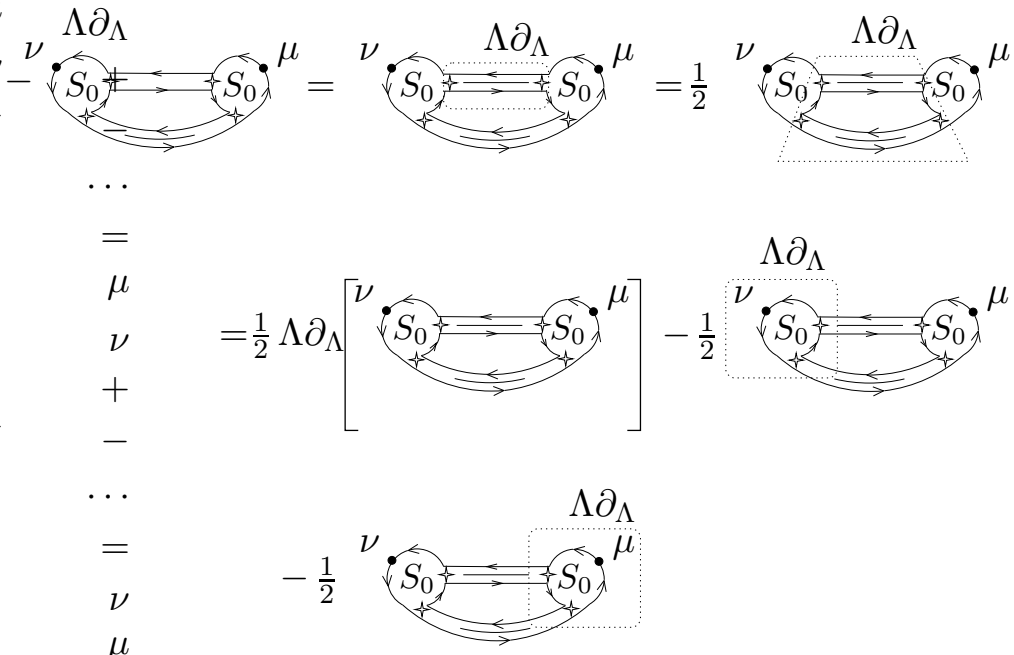

Figure 20: Evaluating the 'smiling frog' diagrams.

Again, making use of the effective propagator relations (6.12,6.13), many cancellations occur. Thus the last two terms on the first line of fig. 13 (and its $p_{\mu} \mapsto-p_{\nu}$ partner) result in cancelling the mixed $\hat{S}-S_{0}$ terms as illustrated in fig. 21. Moreover, the $S_{0}$ part of $\Sigma_{0}^{A F F}$, the last term left in (8.3), is cancelled by the first term on the second line of fig. 13, as can be seen from fig. 22 (after adding the $p_{\mu} \mapsto-p_{\nu}$ partner). The last two terms

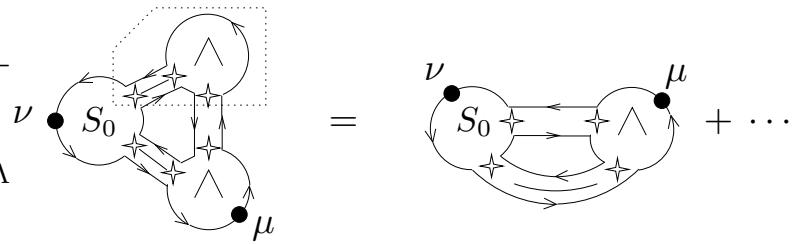

Figure 21: Cancellation of the 'winking smiling frog' diagrams.

in fig. 13 result in diagrams of the form shown in fig. 23. These cancel two corresponding terms generated by fig. 12, where the $S_{0}$ three-point vertex is replaced by the $\hat{S}$ three-point vertex. The cancellation occurs because the top lobe in fig. 23 is already $O\left(p^{2}\right)$, and thus in the bottom lobe, by (3.35) and equality of $S_{0}$ and $\hat{S}$ two-points, what actually counts is

$$
S_{0 \mu R S}^{A F F}(0, k,-k)=\partial_{\mu}^{k} \hat{S}_{R S}^{F F}(k)=\hat{S}_{\mu R S}^{A F F}(0, k,-k) .
$$




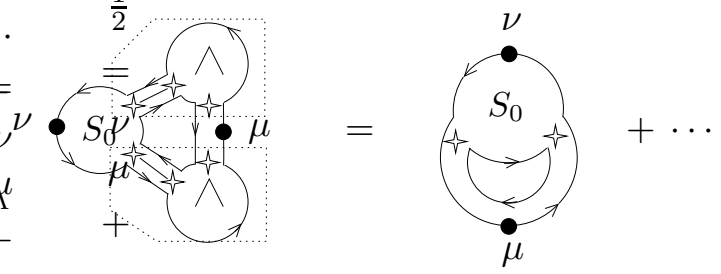

Figure 22: One of a pair cancelling the $S_{0}^{A F F}$ term in fig. 11.

After combining via $p_{\mu} \leftrightarrow-p_{\nu}$, we are left with just the diagram generated by the first

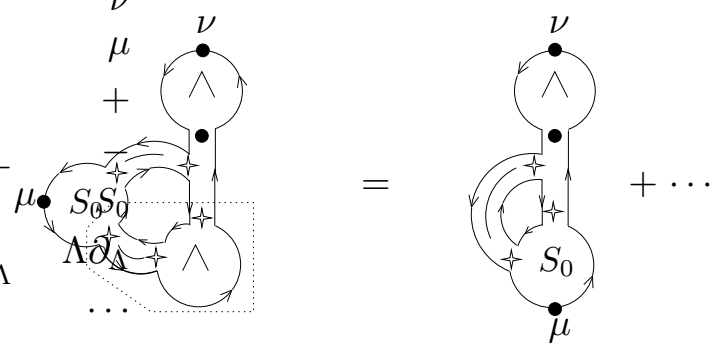

Figure 23: Further diagrams from fig. 13.

term in fig. 13, namely fig. 24, all the other terms from fig. 13 having been dealt with. It

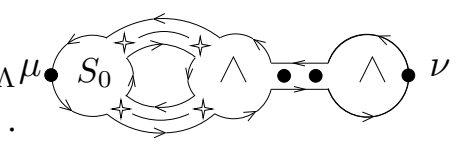

Figure 24: A potentially universal term generated by fig. 13 .

is easy to see by gauge invariance that this is potentially universal, i.e. depends only on two-point seed action vertices and the zero-point kernels. (In fact, just as in fig. 16, this contains $c_{0}^{\prime}$ and must combine to give a vanishing contribution.)

The only remaining terms to be processed are those generated by the first four diagrams on the last line of fig. 12. We easily see however that up to the gauge remainders which as above we set aside, all these correspond to wine-biting-their-tail diagrams and are thus annihilated by (2.37). If we ignore this constraint then, on tidying up using the coincident line identities [10, 11]

$$
\begin{aligned}
\dot{\Delta}_{\mu, R, A \alpha}^{A, F, F A}(p ; k ;-k,-p) & =-\dot{\Delta}_{\mu R, A \alpha}^{A F, F A}(p, k ;-k,-p)-\dot{\Delta}_{R \mu, A \alpha}^{F A, F A}(k, p ;-k,-p), \\
\dot{\Delta}_{, \mu R, A \alpha}^{, A F, F A}(; p, k ;-k,-p) & =\dot{\Delta}_{R \mu, A \alpha}^{F A, F A}(k, p ;-k,-p),
\end{aligned}
$$

etc. , we get a contribution to the integrand in $(8.3)$ of the form:

$$
2 \hat{S}_{\nu \alpha}^{A A}(p) \dot{\Delta}_{\mu R, R \alpha}^{A F, F A}(0, k ;-k, 0) .
$$


Now, since there is no $\dot{\Delta}^{\mathcal{C} \mathcal{A}}$ kernel, we have by (2.34):

$$
\begin{aligned}
\dot{\Delta}_{\mu R, R \alpha}^{A F, F A}(0, k ;-k, 0) & =\dot{\Delta}_{\mu \alpha}^{A B, B A}(0, k ;-k, 0) \\
& =\dot{\Delta}_{\mu \alpha}^{A A, A A}(0, k ;-k, 0) .
\end{aligned}
$$

By replacing $F$ as described below (8.3), we see by the same argument that there is no corresponding $C$ sector contribution, but by (8.14), there is an equal and opposite $A$ sector contribution. Thus the wine-biting-their-tail diagrams exactly cancel here in any case, as already explained below (8.3).

Collecting the results, we have

$$
\begin{aligned}
& -4 \beta_{1} \square_{\mu \nu}(p)+O\left(p^{3}\right)=N \int \frac{d^{4} k}{(2 \pi)^{4}}\left\{\Lambda \partial _ { \Lambda } \left[2 \Delta_{S R}^{F F}(k) S_{0 \mu \nu R S}^{A A F F}(p,-p, k,-k)\right.\right. \\
& \left.-\Delta_{S R}^{F F}(k) \Delta_{T U}^{F F}(k-p) S_{0 \mu T S}^{A F F}(p, k-p,-k) S_{0 \nu R U}^{A F F}(-p, k, p-k)\right] \\
& -\frac{4}{\Lambda^{2}} c_{0}^{\prime} \square_{\nu \alpha}(p)\left[\Delta_{S R}^{F F}(k) \partial_{\mu}^{k} \partial_{\alpha}^{k} \hat{S}_{R S}^{F F}(k)-\Delta_{R U}^{F F}(k) \Delta_{S T}^{F F}(k) \partial_{\mu}^{k} \hat{S}_{R S}^{F F}(k) \partial_{\alpha}^{k} \hat{S}_{T U}^{F F}(k)\right] \\
& +4 \dot{\Delta}_{T U}^{F F}(k) k_{U}^{\prime} k_{R} \hat{S}_{\mu \nu R T}^{A A F F}(p,-p, k,-k)+4 \dot{\Delta}_{\mu, T U}^{A, F F}(p ;-k, k-p) k_{T}^{\prime} k_{R} \hat{S}_{\nu R U}^{A F F}(-p, k, p-k) \\
& -4 S_{0 \nu R U}^{A F F}(-p, k, p-k) \hat{S}_{\mu T M}^{A F F}(p, k-p,-k) \Delta_{T U}^{F F}(p-k) \dot{\Delta}_{M N}^{F F}(k) k_{N}^{\prime} k_{R} \\
& -2 S_{0 \mu R T}^{A F F}(p, k-p,-k)\left[2 \dot{\Delta}_{\nu, U R}^{A, F F}(-p ; p-k, k)(-k)_{U}^{\prime}(-k)_{T}\right. \\
& \left.-\dot{\Delta}_{\nu, U V}^{A, F F}(-p ; p-k, k)(-k)_{U}^{\prime}(-k)_{T}(k-p)_{V}^{\prime}(k-p)_{R}\right] \\
& -8 \square_{\nu \alpha}(p) \dot{\Delta}_{U}^{F, A B}(k ; 0,-k) k_{\alpha}^{\prime} k_{R} \partial_{\mu}^{k} \hat{S}_{R T}^{F F}(k) \Delta_{T U}^{F F}(k) \\
& \left.-8 \square_{\nu \alpha}(p)(-k)_{\alpha}^{\prime}(-k)_{R} \dot{\Delta}_{\mu R}^{A F, B A}(0, k ;-k, 0)\right\}
\end{aligned}
$$

The first two lines contain the total $\Lambda$ derivative terms. The next line contains the only potentially universal terms so far. There then follows in the order generated above, all the gauge remainder terms ${ }^{19}$ in particular the last term is all that is left from the diagrams above that generated wine-biting-their-tail diagrams.

Note that up until now, all the essential steps have been performed at a totally diagrammatic level. ${ }^{20}$ Indeed it is far more efficient and elegant to do so, not the least because the diagrams make very clear the relations due to cyclicity, and charge conjugation (a.k.a. mirror symmetry), and are already identical if the algebraic expressions are equal after relabelling the loop momentum. It is possible to evaluate the gauge transformations in (8.15) also at this level [10, 11], so it may be possible to push these diagrammatic techniques further. However we now have to take account of the differences between the $A, C$ and $F$ sectors. In this paper, we will pursue the remainder of the analysis at the level of equations.

\footnotetext{
${ }^{19}$ Terms related by charge conjugation, $p_{\mu} \leftrightarrow-p_{\nu}$, and relabelling the loop momentum, have been combined.

${ }^{20}$ See ref. 31] for some of the algebraic expressions.
} 


\subsection{The total $\Lambda$ derivative contribution}

We start by computing the $O\left(p^{2}\right)$ part of the first two lines in 8.15). These two terms seem to have the clearest physical interpretation. They both depend only on effective propagators and the effective action $S$ (which, unlike the seed action, can be expected to contain the real physics [18]). Their diagrams, $c f$. fig. 14 and fig. 20, are actually just the usual tadpole and self-energy Feynman graphs respectively. From the translations below (8.3) we see that the $A$ sector looks as though it is in Feynman gauge (although ghosts are missing):

$$
\begin{aligned}
N \Lambda \partial_{\Lambda} \int \frac{d^{4} k}{(2 \pi)^{4}}\left[-2 \Delta_{k}^{A A} S_{0 \mu \nu \alpha \alpha}^{A A A A}(p,-p, k,-k)\right. & \\
& \left.+\Delta_{k}^{A A} \Delta_{k-p}^{A A} S_{0 \mu \alpha \beta}^{A A A}(p, k-p,-k) S_{0 \nu \beta \alpha}^{A A A}(-p, k, p-k)\right]_{O\left(p^{2}\right)}^{A A},
\end{aligned}
$$

whilst the $F$ and $C$ sectors via (6.1) and (6.4) give copies with $\sim \Lambda$ massive effective propagators, that are the expected Pauli-Villars regularising terms. Indeed if the five dimensional notation is expanded using (6.5), we see that apart from the relative minus sign, the $B$ sector looks identical in form to (8.16). Similarly, since the sign in (6.10) cancels on translating the vertices back to $A A D D$ and $A D D$ form, the $D$ sector has identical appearance, but opposite sign, to the $C$ sector. Since we have ensured that at high momentum $k$, the $B(D)$ terms do actually equal the $A(C)$ terms, we see that the regularisation is incorporated as required. Finally, the overall $\Lambda$ derivative is just as one would expect to convert the $\ln \Lambda$ divergent result to a contribution to the $\beta$ function 18.

As shown in (8.16), we now exchange the order of $\Lambda \partial_{\Lambda}$ and the $k$ integral. Pulling out the $O\left(p^{2}\right)$ part, the integral is actually dimensionless. Since the only explicit scale is $\Lambda$, if the integral were convergent it would have to be a constant, thus (8.16) combined with the $B, C, D$ sectors, would vanish. In fact the momentum integral is not well defined only because the second term in the $A$ sector has an infrared divergence. We can still keep $\Lambda \partial_{\Lambda}$ outside if we use the standard trick of introducing an infrared cutoff $k>\epsilon$ in this term, taking $\epsilon \rightarrow 0$ at the end. Now this term, and this term only, depends on $\Lambda$, through $\Lambda / \epsilon$. Therefore it suffices to analyse its infrared behaviour, replacing $\Lambda \partial_{\Lambda}$ with $-\epsilon \partial_{\epsilon}$.

Note that the $S_{0}$ vertices have Taylor expansions in small momenta, $c f$. sec. 2.2, and the $B, C$ and $D$ effective propagators (6.1), (6.4), are regular as $k \rightarrow 0$, whilst the $A$ effective propagator (6.2) has an infrared double pole. Thus only in the $A$ sector is there a problem. The result is integrable in the first term in (8.16) but not in the second, once expanded to order $p^{2}$. However, whilst $\partial_{\Lambda}$ is inside the integral there is actually no infrared divergence, it being ameliorated via (5.6) or the flow equations for the three-point vertices, equivalently as we derive below, because the only terms in the three-point vertices that contribute are independent of $\Lambda$. One can then confirm that the above limit $\epsilon \rightarrow 0$ gives the same answer. Of course the same answer is also obtained by using dimensional regularisation or by converting this part to a total derivative in $k^{2}$ as e.g. in ref. 18.

If we had not taken care to exclude the logarithmic classical divergences in sec. 7.1, it is at this point that we would have picked up extra contributions since the $B, C, D$ parts of the integral can then be functions of $\Lambda / \mu$. Worse, we have confirmed that if these parts 
are evaluated first, using here $\partial / \partial \Lambda \equiv-\partial / \partial \mu$, the resulting $k$ integrals are non-universal, as may be expected from the general arguments in sec. 7. However, in ref. [11, where very similar classical $\mu$ dependent terms were not excluded, the right answer was obtained by keeping an upper limit $\Lambda_{0}$ in the $\Lambda$ integrals as in (7.3), and in fact for these terms sending $\Lambda_{0} \rightarrow \infty$ only after the $k$ integral had been performed. It thus appears that if the logarithmic classical divergences are not excluded, then the result actually depends on the order in which the ultraviolet limits on the $\Lambda$ and $k$ integrals are performed.

Returning to the main analysis, we note that we are interested in expanding in $p$ and then in $k . S_{0 \mu \nu \lambda}^{A A A}$ is regular in small momenta and the lowest order term in its momentum expansion, with one momentum, is fixed uniquely by (3.5) to be the standard Feynman gluon vertex. If we take the $p$ part in both three-points in (8.16), this already saturates the order $p^{2}$ required. Furthermore, we then must take only the $1 / 2 k^{2}$ parts of the effective propagators in order to get an infrared divergence. If we take a $k$ part from the three-point vertices, then in order to maintain an infrared divergence we must use one of the more divergent terms from the $p$ expansion of $1 / 2(k-p)^{2}$, thus again saturating the expression at $O\left(p^{2}\right)$. Thus we see that the only part that contributes to the total $\Lambda$ derivative terms in (8.15), is universal [as a consequence of (3.5)], and precisely of the same form as the standard gluonic self-energy term in Feynman gauge. We have

$$
\begin{aligned}
N \epsilon \partial_{\epsilon} \int_{\epsilon} \frac{d^{4} k}{(2 \pi)^{4}}\left\{\frac{1}{k^{4}} \partial_{\beta}^{p} \square_{\mu \alpha}(p) \partial_{\beta}^{p} \square_{\nu \alpha}(p)+\left[4 \frac{(k \cdot p)^{2}}{k^{8}}-\frac{p^{2}}{k^{6}}\right] \partial_{\mu}^{k} \square_{\alpha \beta}(k) \partial_{\nu}^{k} \square_{\alpha \beta}(k)\right. \\
\left.+2 \frac{k \cdot p}{k^{6}}\left[\partial_{\mu}^{k} \square_{\alpha \beta}(k) \partial_{\beta}^{p} \square_{\alpha \nu}(p)+\partial_{\beta}^{p} \square_{\mu \alpha}(p) \partial_{\nu}^{k} \square_{\alpha \beta}(k)\right]\right\} \\
=\frac{N}{(4 \pi)^{2}}\left\{\frac{19}{3} p^{2} \delta_{\mu \nu}-\frac{22}{3} p_{\mu} p_{\nu}\right\},
\end{aligned}
$$

where on the left hand side we used (8.10) to evaluate $S_{0 \mu \alpha \beta}^{A A A}(p,-p, 0), S_{0 \mu \alpha \beta} A A A(0, k,-k)$, etc. , and substituted $S_{0 \mu \nu}^{A A} \sim 2 \square_{\mu \nu}$, cf. (4.3,4.4), and the right hand side follows after averaging over $k$ directions and expressing as a radial $k$ integral.

As expected of a universal term, (8.17) does not, apparently, depend on the regulating $C$ and $F$ sectors. But despite this, and the apparently clear physical interpretation, it is not transverse on its own as would be required by gauge invariance. This result seems all the more surprising once one notes that actually at a formal level the $A$ sector is gauge invariant on its own [10] (and as we will confirm, the other $A$ sector terms in (8.15) are already transverse). Indeed, taking care to keep the $\Lambda$ derivative inside the (8.16) integral (so there is no problem with infrared divergences), contracting with $p_{\mu}$ and $-p_{\nu}$, then using (3.35), (4.3,4.4) and (6.2), and shifting $k$ in some terms (discarding one odd in $k$ ), ${ }^{21}$ one finds that the longitudinal part of (8.16) is

$$
2 N \int \frac{d^{4} k}{(2 \pi)^{4}} \Lambda \partial_{\Lambda} \frac{p_{\beta} \square_{\beta \nu}(k) p_{\nu}}{k^{2}(k-p)^{2}},
$$

which obviously vanishes since the remaining terms have no $\Lambda$ dependence. Of course these manipulations do not make sense without ultraviolet regularisation. The apparent

\footnotetext{
${ }^{21}$ See refs. [31, 10, 11] for further comments pertinent to here and above.
} 
independence of (8.17) on the $C$ and $F$ sectors is illusory since the derivation of 8.17) from (8.16) is only legitimate if such sectors exist with the property that they cancel its ultraviolet divergences whilst not adding any new infrared ones. Taking into account the other sectors, one finds that the $C$ sector also gives a purely transverse contribution, but the $F$ sector yields a longitudinal part, which is precisely the one in (8.17).

The full understanding of the apparent Feynman gauge in the total derivative term (8.17) is closely related. It comes from the absence of longitudinal terms in the RG equation, which were not included, as by supergauge invariance they could be exchanged for $\mathcal{C}$ commutators, $c f .(3.15)$. Had we introduced them, e.g. as the term

$$
\frac{1}{2} \nabla_{\mu} \cdot \frac{\delta S}{\delta \mathcal{A}_{\mu}}\left\{\dot{\Delta}_{L}\right\}_{\mathcal{A}} \nabla_{\nu} \cdot \frac{\delta \Sigma_{g}}{\delta \mathcal{A}_{\nu}}
$$

in (3.2) [and correspondingly in (3.3)], the $A$ effective propagator would change to include the characteristic longitudinal dependence in general gauge, with $^{22}$

$$
\Delta_{L}(p, \Lambda)=\frac{1}{p^{4}}\left[\frac{1}{\xi}-1+O\left(x^{2}\right)\right]
$$

parametrising it, generalising the usual gauge fixing parameter dependence. Such a change would of course be compensated for by similar changes in the $B$ and $D$ effective propagators, via (3.15) or (4.5). However, unlike in a gauge fixed theory, the two-point classical action vertices would remain as (4.3), (4.6) and (4.7), gauge invariant and completely independent of the introduction of $\Delta_{L}$. The calculation would simply have been rearranged, by finessing some longitudinal parts into the $F$ sector.

Finally, let us make a trivial, but important, observation. Although (8.17) is precisely the $\ln (\Lambda / \epsilon)$ contribution from the standard gluonic self-energy term in Feynman gauge, it is here a contribution to the Wilsonian effective action, not a contribution to the $S$ matrix. Feynman's unitarity arguments for the existence of ghosts [35] cannot be directly applied to vertices of the Wilsonian effective action. Of course, if we wished to talk about on-shell gluons, we would have to gauge fix and introduce ghosts, but that is not what we are talking about. As already discussed, here the ghostly contributions are replaced by shadows from the regularisation sector.

\subsection{The gauge remainders}

We next turn to the gauge remainders, the fourth line onwards in (8.15). As well as the $F$ sector shown, we also have the $A$ sector contributions which we consider first. Recall that they come with opposite sign and are translated as described at the beginning of this section. Making use of (3.35) and the same sort of simplifications as in the previous subsections, many terms cancel out, including some that would otherwise not be potentially universal. We are left with purely transverse contributions: an ultraviolet divergent contribution to the integrand ${ }^{23}$

$$
\frac{8 c_{0}^{\prime}}{\Lambda^{2} k^{2}} \square_{\mu \alpha}(p)\left(\delta_{\alpha \nu}-k_{\alpha} k_{\nu} / k^{2}\right)
$$

\footnotetext{
${ }^{22}$ The $x=p^{2} / \Lambda^{2}$ term is missing by (3.11), because (8.19) must be analytic in $p c f$. sec. 2.2.

${ }^{23}$ Clearly, by Lorentz invariance of the $k$ integration, this is transverse on $\nu$ also.
} 
which will be combined later with the potentially universal terms on the third line of 8.15) for the reason given below 8.9 ), and after averaging over $k$ directions, a term

$$
-\frac{2 N}{\Lambda^{4}} \square_{\mu \nu}(p) \int \frac{d^{4} k}{(2 \pi)^{4}}\left(5 c_{k}^{\prime} \Lambda^{2} / k^{2}+c_{k}^{\prime \prime}\right)=\frac{N}{2 \pi^{2}} \square_{\mu \nu}(p),
$$

where we have used the fact that, after casting as an $x=k^{2} / \Lambda^{2}$ integral, the integrand is a total derivative, vanishing at $x=\infty$ and universal at $x=0$ by $c(0)=1$.

Evaluating the $F$ sector using (6.11) and similar simplifications to above, but keeping the compact 5 dimensional notation, already many terms cancel out, again in particular all terms that would otherwise not be potentially universal. This time the remaining terms are all clearly transverse except one:

$$
\left.4 \Delta_{S N}^{F F}(k) k_{N}^{\prime}(k-p)_{\nu}^{\prime} \hat{S}_{\mu S}^{B F}(k)\right|_{O\left(p^{2}\right)}
$$

After some algebra, and including the integral in (8.15), this takes the form

$$
-\frac{2}{3} \frac{N}{(4 \pi)^{2}}\left(p^{2} \delta_{\mu \nu}+2 p_{\mu} p_{\nu}\right) \int_{0}^{\infty} d x g^{\prime}(x)\left(x^{3} f^{\prime}(x)\right)^{\prime},
$$

where $f$ and $g$ were defined in (6.14). Substituting $g$ in terms of $f$ and integrating by parts this may be cast as a total derivative. Using $\tilde{c}(0)=c(0)=1$ and for example (2.11), it evaluates to

$$
\frac{1}{3} \frac{N}{(4 \pi)^{2}}\left(p^{2} \delta_{\mu \nu}+2 p_{\mu} p_{\nu}\right)
$$

Although it is not transverse, added to (8.17), it results in a transverse contribution, namely $20 N / 3(4 \pi)^{2} \square_{\mu \nu}(p)$. In view of the comments below (8.18), we should expect to find such a non-transverse correction lurking in the $F$ sector.

The transverse supergauge remainder terms yield a contribution to the integrand of (8.15) of

$$
-\frac{8}{\Lambda^{2}} c_{0}^{\prime} \square_{\mu \alpha}(p)\left[k_{\alpha}^{\prime} k_{\nu}^{\prime}+\partial_{\nu}\left(k_{\alpha} / k^{2}\right)\right]
$$

to be compared with (8.21) and similarly saved for later, and a number of terms which after some algebra turn into a total derivative in $x$, yielding

$$
-\left.\frac{2 N}{(4 \pi)^{2}} \square_{\mu \nu}(p)\left(x c^{\prime}-4 c\right) x^{2} f^{2}\right|_{0} ^{\infty}
$$

(where $f$ and $c$ are evaluated at $x$ ). Considering again the behaviour of $c$ and $\tilde{c}$ at the boundaries, we see that the result vanishes.

We are left only to evaluate the terms proportional to $c_{0}^{\prime}$. Before we do so, we note that the total contribution to (8.15) from the other terms, namely (8.17), (8.22) and 8.25) sum to $44 N / 3(4 \pi)^{2} \square_{\mu \nu}(p)$, i.e. already yielding the famous result

$$
\beta_{1}=-\frac{11}{3} \frac{N}{(4 \pi)^{2}}
$$




\subsection{The $c_{0}^{\prime}$ terms}

As we have already anticipated, all the $c_{0}^{\prime}$ terms must sum to zero. Together with 8.21) and (8.26), we also have the three sectors contributions from the third line of 8.15$)$. This term can be simplified in a way that can be mapped also to the $C$ and $A$ sectors. Thus, suppressing the $F$ superscripts and the $k$ momentum arguments:

$$
\begin{aligned}
& \Delta_{S R} \partial_{\mu} \partial_{\alpha} \hat{S}_{R S}-\Delta_{R U} \Delta_{S T} \partial_{\mu} \hat{S}_{R S} \partial_{\alpha} \hat{S}_{T U} \\
& =\partial_{\mu}\left(\Delta_{R S} \partial_{\alpha} \hat{S}_{R S}\right)-\partial_{\mu} \Delta_{R S} \partial_{\alpha} \hat{S}_{R S}-\Delta_{R U} \Delta_{T S} \partial_{\mu} \hat{S}_{R S} \partial_{\alpha} \hat{S}_{T U} \\
& \equiv \partial_{\mu}\left(\Delta_{R S} \partial_{\alpha} \hat{S}_{R S}\right)-\Delta_{R U} \partial_{\alpha} \hat{S}_{R S} \partial_{\mu}\left(k_{U}^{\prime} k_{S}\right)-k_{T}^{\prime} k_{R} \partial_{\mu} \Delta_{T S} \partial_{\alpha} \hat{S}_{R S} \\
& =\partial_{\mu}\left(\Delta_{R S} \partial_{\alpha} \hat{S}_{R S}\right)-2 k_{\alpha}^{\prime} k_{\mu}^{\prime}-2 \partial_{\alpha} k_{\mu}^{\prime}
\end{aligned}
$$

This holds in all sectors using the maps at the beginning of this section. (To see this, rewrite the third term of the second line to contain $\partial_{\mu}\left(\Delta_{R U} \hat{S}_{R S}\right)$ plus remainder, and use 6.12, 6.13), and $k \mapsto-k$. In the third line, third term, transfer the $\partial_{\alpha}$ to $k_{R}$, using (6.8), equivalently (4.2), and use $\partial_{\alpha} k_{R}=\delta_{R \alpha}$, which holds in both $F$ and $A$ sectors. Finally in this term form $\partial_{\mu}\left(S_{R S} \Delta_{S T}\right)$ and simplify similarly, noting that since it is a function only of $k$ it is automatically $\alpha \leftrightarrow \nu$ symmetric.)

By dimensions and Lorentz invariance $\Delta_{R S} \partial_{\alpha} \hat{S}_{R S}=2 k_{\alpha} F(x) / \Lambda^{2}$ for some function $F\left(x=k^{2} / \Lambda^{2}\right)$, with corresponding expressions in the $A$ and $C$ sectors. Indeed by (6.1), we have for the $C$ sector, $F=\frac{d}{d x} \ln (2 \lambda+x / \tilde{c})$; by (6.2) and (4.3), we have $F=3 \frac{d}{d x} \ln (x / c)$ in the $A$ sector; and finally in the $F$ sector, using (4.1), (4.6), (6.10) and (6.4),

$$
F=\frac{d}{d x} \ln (x / c+2 / \tilde{c})+\frac{d}{d x} \ln \left(x / \tilde{c}+2 c / \tilde{c}^{2}\right)-\frac{c}{x \tilde{c}+2 c}-\frac{\tilde{c}^{2}}{x \tilde{c}+2 c} \frac{d}{d x} \frac{c}{\tilde{c}^{2}} .
$$

Including the multiplicative factors in 8.15$)$, the first term of 8.29 ) thus gives the surface contribution:

$$
-\left.\frac{N}{4 \pi^{2}} c_{0}^{\prime} \square_{\mu \nu}(p) x^{2} F\right|_{0} ^{\infty} .
$$

Summing this contribution across all sectors (remembering the relative minus sign for $A$ and $C$ ), one finds no contribution from the $x=0$ boundary in any sector, whilst the otherwise divergent $x=\infty$ terms cancel exactly between the fermionic and bosonic sectors.

This leaves just the second and third terms of (8.29). Incorporating the extra factors in (8.15), we see that the second term cancels the first in (8.26), whilst the remaining terms combine to an ultraviolet finite total derivative $2 \partial_{\mu}\left(k_{\alpha} / k^{2}-k_{\alpha}^{\prime}\right)$. Comparing with the above structure and using (6.15), we see trivially that this integrates to (8.31) with $F=1 / x-f$, and thus vanishes. Finally, recalling the relative minus sign, we see that the $A$ sector translation of the second and third terms in (8.29) exactly cancel (8.21). We have thus shown that all the $c_{0}^{\prime}$ terms disappear, as expected.

\section{Conclusions}

We have proposed a technique which allows manifestly gauge invariant and universal computations to be performed, directly in the continuum. No gauge fixing or ghosts are 
required, thus avoiding completely the problem of Gribov copies [13]. The full power and beauty of local gauge invariance is clear and central to the whole calculation. Moreover, renormalization group properties are built in from the beginning. The calculation proceeds very economically, by confirming and exploiting the independence of physical quantities on the details of the regularisation.

At its heart, lies the successful combination of gauge invariance with the introduction of a real effective cutoff [16]. This has long been an outstanding problem, as any straightforward division of momenta into large and small, according to some effective scale $\Lambda$, is not preserved by gauge transformations. (Explicitly, under a gauge transformation of some matter field $\phi(x) \rightarrow \Omega(x) \phi(x)$, momentum modes $\phi(p)$ are mapped to a convolution with the modes from $\Omega$.) Its solution allows us to use Wilson's insight [2, 8] making renormalization properties, normally subtle and complicated, trivially clear and straightforward.

This continuum cutoff is simply spontaneously broken $S U(N \mid N)$ gauge theory with covariant higher derivatives. Sketched at the start of sec. 2 (for a complete analysis, see [16]), it includes two copies of the $S U(N)$ gauge field, $A_{1}$ and $A_{2}$, and a pair of wrong statistics gauge fields $B, \bar{B}$. A superscalar field is added to cause spontaneous symmetry breaking down to the bosonic $S U(N) \times S U(N)$, giving masses of order the cutoff to all fields but $A_{1}$ and $A_{2}$. Depending on the representation chosen, one also encounters a $U(1)$ connection, $\mathcal{A}^{0}$. This latter, however, does not appear anywhere in the action provided all interactions are of the form $\operatorname{str}(\mathcal{A} \times$ commutators $)$, more generally provided a no- $\mathcal{A}^{0}$ shift symmetry $\delta \mathcal{A}_{\mu}=\lambda_{\mu} 1$ is respected. Such a shift symmetry, which amounts to dynamically defining the coset space $S U(N \mid N) / U(1)$, plays an important rôle, ensuring that our flow equation, (3.1), is indeed gauge invariant [cf. eqs. (3.12)-(3.14)]. We can already see that it will play an even more central rôle in the future [1].

This regularisation fits very well within the effective action framework. A manifestly gauge invariant flow equation for the Wilsonian effective action, $S$, has been written down, (3.1), which leaves the partition function - and hence the physics derived from it - unchanged. This is achieved by demanding that the Boltzmann measure, exp $-S$, flow into a total derivative.

Solutions for $S$ can then be found directly in terms of renormalized quantities, without specifying a bare action. Note that almost any approximation can be considered without spoiling this property [5].

As discussed in sec. 2.2, since the partition function is left unchanged, it is possible to interpret our results at different scales $\Lambda$. The fact that locality is correctly incorporated, for example, is best understood through the $\Lambda=\infty$ limit, recalling that the effective action is Taylor expandable to all orders in momenta (this requirement of "quasilocality" [10], is tantamount to demanding that each infinitesimal RG step be free from infrared singularities) and that $\Lambda$ is the only explicit scale parameter to appear in the action, thus implementing the concept of a self-similar flow [27]. Again it is at $\Lambda \rightarrow \infty$ that we see we are indeed describing $S U(N)$ gauge theory: massive fields are actually infinitely massive and $A_{2}$ decouples, as ensured by the Appelquist-Carazzone theorem [21].

Besides manifest gauge invariance, another important step is the exploitation of the freedom coming from scheme independence [8]: not only did the seed action $\hat{S}$ incorporate 
the gauge invariant regularisation and allow the spontaneous symmetry breaking [see comments below (3.10)], but also we could choose it such that the minimum of the potential would flow with $\Lambda$ (cf. (3.16) and below) and such that logarithmic classical divergences would be completely absent (see sec. 7.1). This latter property guarantees the universality of the one-loop Yang-Mills $\beta$ function ( $c f$. sec. 7 ). By further fashioning $\hat{S}$, these properties extend to two-loop order and higher [1]. In a sense the generalised exact RG framework [9, 8] gives us the freedom to create 'designer field theories' in the regularisation sector, tailored to our purpose.

The seed action (not bare action) therefore represents the details put in by hand; the physics is naturally encoded in the effective action. Hence, physical quantities must be independent of the choice of $\hat{S}$ [8], as our tests here, and earlier [18], have shown. Note: we do not pick a specific $\hat{S}$. Apart from the constraints above, it turns out to be very advantageous to keep it as general as possible. Since the final result must be independent of its detailed form, any simplification has to occur before we look into any of its vertices. Similarly, we do not specify what covariantisation we use. All we need is to name the vertices ( $c f$. sec. 2.4).

We do introduce some further restrictions purely for convenience. Thus we determine the RG kernels $\dot{\Delta}$ in such a way that after spontaneous symmetry breaking, the two-point classical effective action vertices can be set equal to those of the seed action. This greatly simplifies the flow equations for higher point interactions, $c f$. sec. 3 [ [9, 10]. Actually, the two-point seed action vertices are not as general as they can be, $c f$. sec. 4 . It would not be difficult to work with completely general versions however, leaving no restrictions except those placed by smoothness, symmetries (including broken supergauge invariance) and limits on their ultraviolet behaviour, cf. e.g. (2.11). Very little would change in the calculation right up to the final stages in secs. 8.3 and 8.4 .

Last but not least, the diagrammatic interpretation introduced in sec. 3.2 (generalising that of [9, 10, 11] to finite $N$ ) turned out to be very useful. Already at the level of diagrams the big potential of the method comes out, e.g. making cancellations among higher order seed action vertices self evident.

In view of the novelty of the construction, it is desirable to test the formalism first. We have computed for the first time at finite $N$ without fixing the gauge, the one-loop $\beta$ function for $S U(N)$ Yang-Mills theory, verifying the standard result, and confirming its universality with respect to regularisation scheme.

Let us briefly recapitulate the simple steps that form the calculation, as discussed in detail in sec. 8. From the renormalization condition, eq. (3.5), we can write down an algebraic equation for the one-loop beta function, $\beta_{1}$ ( $c f$. Fig. 11), which receives contributions, equal in form, from all the sectors of the theory, $A, C$, and $F$. Introducing integrated kernels and integrating by parts the diagram containing the effective action vertex with the highest number of points, leaves us with a total $\Lambda$-derivative plus the $\Lambda$-derivative of this vertex (see fig. 14). Then using the flow equation and the relation between integrated kernels and two-point functions, as in (6.1) and (6.3), to simplify the diagrams obtained so far, we can eliminate the higher point vertices. Repeating the above steps several times over, we get to the point where there is no dependence left on the details of the covariantisation 
or on the seed action. ${ }^{24}$

This last step follows either because we are left with expressions containing only twopoint vertices and the corresponding zero point kernels, which are then shown to be universal by casting them as total derivatives in momentum space, where they depend only on ultraviolet limits or in the infrared on the renormalization condition (3.5), or, as in the total $\Lambda$-derivative term, because the result can be seen explicitly to depend only on vanishing momenta where again the dependence becomes universal as a consequence of (3.5).

Some comments are in order. As emphasised earlier, central to the method is the use of manifest gauge invariance. It results in two sets of "trivial" Ward identities, derived in sec. 3.4, corresponding to the (bosonic) fermionic parts of the (un)broken gauge invariance. The broken Ward identities, in particular, cause $B$ and $D \sigma$ to rotate into one another. This led to the important realisation that technically they should be tied together as elements of a five dimensional vector, $F_{M}, c f$. (6.5). As well as simplifying greatly the calculations in this sector, it also casts them in a form which is virtually isomorphic with the computations in the $A$ and $C$ sectors.

The calculation has been carried out in dimension $D=4$, even though to rigorously define the regularisation to all orders, one should preregularise, e.g. by working in general dimension $D$ and taking the limit $D \rightarrow 4$ at the very end [16]. This is necessary in general [9] because finiteness is achieved by adding separately divergent pieces together, namely the $F$ versus $A$ and $C$ sectors, as is typical of Pauli-Villars type regularisations. However at one loop, it is only necessary to preregularise the four-point $\mathcal{A}$ vertex; the two-point vertex (which is what we compute) is well defined without preregularisation, providing only that global $S U(N \mid N)$ invariance is kept manifest throughout the computation [16].

We did not do this, since we broke the fields $\mathcal{A}$ and $\mathcal{C}$ into their fermionic and bosonic parts. (Had we worked with the full superfields we would not have been able to (anti)commute $\sigma$. This leads to a substantial increase in the number of different vertices required for the computation.) But, by performing the calculation in the $F$ sector and then simply mapping the results to the $A$ and $C$ sectors using the insight above, we performed the calculation in the same way for all sectors, achieving the same effect as would be obtained by keeping the global $S U(N \mid N)$ invariance manifest. ${ }^{25}$

The final result, (8.28), comes almost entirely from the $A$ sector, except for the nontransverse term, (8.25), which however is needed to make the whole thing transverse. On the one hand, we should not be surprised if the whole contribution had come from just the $A$ sector. After all, the one-loop $\beta$ function can be cast as the derivative of the cutoff on an infrared divergent integral. If the fields are massive (viz. $B, C, D$ ), their masses act instead as the infrared regulator and thus they give vanishing contribution [as in the discussion below (8.16)]. On the other hand, the pure gauge sector yields (8.17) plus (8.22), apart from contributions that must vanish eventually e.g. (8.21), and is thus not transverse on its own. As discussed in sec. 8.2, the culprit, (8.17), takes precisely the form of the oneloop gluon self-energy in Feynman gauge, but through an accident of simplicity. We could

\footnotetext{
${ }^{24}$ The calculation in ref. [18] can also be organised according to this iterative method.

${ }^{25}$ We have also checked explicitly that there are no ambiguities related to the limit $D \rightarrow 4$ 31.
} 
have introduced longitudinal terms (8.19) into the flow equation (3.2), giving an apparent general gauge, which by gauge invariance (3.15), finesses longitudinal parts into the $F$ sector. All the while, the effective action would remain oblivious to these rearrangements, and it and all stages in the calculation remain gauge invariant. However in this limited sense, it would appear that the ghost contributions, required in the standard treatment, are here taken over by the $F$ sector.

Finally, although the only application of our method as yet is the calculation of $\beta_{1}$, we expect the procedure to be quite general and, above all, best suited for exploring the non-perturbative domain. It should allow investigations of instantons, renormalons and other controlled non-perturbative effects in a manifestly gauge invariant way.

Generalisations to include space-time supersymmetry and/or fermions seem possible and would open the door to many further investigations, from Seiberg-Witten methods [36] through to fully non-perturbative approximations.

\section{Acknowledgments}

T.R.M. and S.A. acknowledge financial support from PPARC Rolling grant PPA/G/O/2000/00464. It is a pleasure to thank Hugh Osborn and Jean Zinn-Justin for useful discussions.

\section{References}

[1] work in progress.

[2] K. G. Wilson and J. Kogut, Phys. Rept. 12C (1974) 75.

[3] K. G. Wilson, Phys. Rev. D 10 (1974) 2445; in New Phenomena in Subnuclear Physics (Erice '75), ed. A. Zichichi (Plenum Press, New York, 1977); in Recent Developments in Gauge Theories (Cargese '79), ed. G. 't Hooft (Plenum Press, New York, 1980).

A. Hasenfratz and P. Hasenfratz, Nucl. Phys. B 270 (1986) 687.

T. R. Morris, in New Developments in Quantum Field Theory, NATO ASI series 366, (Plenum Press, 1998).

J. Berges, N. Tetradis and C. Wetterich, Phys. Rept. 363 (2002) 233.

C. Wetterich, Phys. Lett. B 301 (1993) 90.

C. Bagnuls and C. Bervillier, Phys. Rept. 348 (2001) 91.

J. Polonyi, hep-th/0110026.

J.-I. Sumi, W. Souma, K.I. Aoki, H. Terao and K. Morikawa, hep-th/0002231.

[4] T. R. Morris, Int. J. Mod. Phys. A 9 (1994) 2411

[5] T. R. Morris, in Yukawa Int. Seminar '97, Prog. Theor. Phys. Suppl. 131 (1998) 395.

[6] See e.g. A. Campbell-Smith and N.E. Mavromatos, Acta Phys. Polon. B29 (1998) 3819.

[7] T. R. Morris, Phys. Lett. B 378 (1996) 213; M. Bonini, M. D'Attanasio and G. Marchesini, Nucl. Phys. B 418 (1994) 81.

[8] J. I. Latorre and T. R. Morris, J. High Energy Phys. 0011 (2000) 004.

[9] T. R. Morris, in The Exact Renormalization Group, Eds Krasnitz et al, World Sci (1999) 1. 
[10] T. R. Morris, Nucl. Phys. B 573 (2000) 97.

[11] T. R. Morris, J. High Energy Phys. 0012 (2000) 12

[12] T. R. Morris, Int. J. Mod. Phys. A 16 (2001) 1899.

[13] V. N. Gribov, Nucl. Phys. B 139 (1978) 1.

[14] H. Neuberger, Phys. Lett. B 183 (1987) 337.

[15] L. Baulieu, A. Rozenberg and M. Schaden, Phys. Rev. D 54 (1996) 7825.

[16] S. Arnone et al., Int. J. Mod. Phys. A17 Vol. 17 (2002) 2283.

[17] A. A. Slavnov, Theor. Math. Phys. 33 (1977) 977; L. D. Faddeev and A. A. Slavnov, Front. Phys. 50 (1980) 1 [Front. Phys. 83 (1990) 1].

[18] S. Arnone, A. Gatti and T. R. Morris, J. High Energy Phys. 0205 (2002) 059.

[19] N. Berkovits, C. Vafa and E. Witten, J. High Energy Phys. 9903 (1999) 018.

[20] I. Bars, in Introduction to Supersymmetry in Particle and Nuclear Physics, eds. Castaños et al. (Plenum, New York, 1984) p. 107.

[21] T. Appelquist and J. Carazzone, Phys. Rev. D 11 (1975) 2856; K. Symanzik, Comm. Math. Phys. 34 (1973) 7.

[22] A. A. Slavnov, Theor. Math. Phys. 13 (1972) 1064; R. W. Lee and J. Zinn-Justin, Phys. Rev. D 5 (1972) 3121.

[23] see e.g. J. Zinn-Justin, "Quantum Field Theory and Critical Phenomena" (1993) Clarendon Press, Oxford.

[24] C. Itzykson and J. B. Zuber, "Quantum Field Theory" (1985) McGraw-Hill, Singapore

[25] F. J. Wegner and A. Houghton, Phys. Rev. A 8 (1973) 401.

[26] T. R. Morris, Phys. Lett. B 329 (1994) 241; Phys. Lett. B 334 (1994) 355; Nucl. Phys. B 458[FS] (1996) 477.

[27] D. V. Shirkov, Theor. Math. Phys. 60 (1985) 778.

[28] P. Hasenfratz and F. Niedermayer, Nucl. Phys. B 414 (1994) 785

[29] G. 't Hooft, Nucl. Phys. B 72 (1974) 461.

[30] J. Polchinski, Nucl. Phys. B 231 (1984) 269.

[31] A. Gatti, "A Gauge Invariant Flow Equation", Ph.D. Thesis.

[32] J. Goldstone, Nuovo Cim. 19 (1961) 154.

[33] M. Bonini, G. Marchesini and M. Simionato, Nucl. Phys. B 483 (1997) 475.

[34] J.M. Pawlowski, Int. J. Mod. Phys. A16 (2001) 2105.

[35] R.P. Feynman, Acta Phys. Polon. 24 (1963) 697.

[36] N. Seiberg and E. Witten, Nucl. Phys. B 426 (1994) 19, Erratum-ibid. B 430 (1994) 485; Nucl. Phys. B 431 (1994) 484. 